\title{
ON THE SCHWARTZ SPACE OF THE BASIC AFFINE SPACE
}

\author{
ALEXANDER BRAVERMAN AND DAVID KAZHDAN
}

\begin{abstract}
Let $G$ be the group of points of a split reductive algebraic group $\mathbf{G}$ over a local field k and let $X=G / U$ where $U$ is the group of $k$-points of a maximal unipotent subgroup of $\mathbf{G}$. In this paper we construct certain canonical $G$-invariant space $\mathcal{S}(X)$ (called the Schwartz space of $X$ ) of functions on $X$, which is an extension of the space of smooth compactly supported functions on $X$. We show that the space of all elements of $\mathcal{S}(X)$, which are invariant under the Iwahori subgroup of $G$ coincides with space generated by the elements of the so called periodic Lusztig's basis, introduced recently by G. Lusztig (cf. [10] and [11]). We also give an interpretation of this space in terms of certain equivariant K-group (this was also done by G.Lusztig - cf. 12]). Finally we present a global analogue of $\mathcal{S}(X)$, which allows us to give a somewhat untraditional treatment of the theory of principal Eisenstein series.
\end{abstract}

\section{INTRODUCTION}

1.1. Let $k$ be a non-arcimedian local field and let $\mathbf{G}$ be a split reductive connected algebraic group over $k$. We will assume also that the derived group $[\mathbf{G}, \mathbf{G}]$ is simply connected. Let $\mathbf{X}=\mathbf{G} / \mathbf{U}$ where $\mathbf{U}$ is a maximal unipotent subgroup of $\mathbf{G}$ defined over $k$. The group $\mathbf{G}$ acts on $\mathbf{X}$ on the left.

In this paper we are going to study the space of functions on $X \stackrel{\text { def }}{=} \mathbf{X}(k)$ (the set of $k$-points of $\mathbf{X})$. Usually one studies the space $\mathcal{S}_{c}(X)$ of locally constant, compactly supported functions on $X$. The group $G=\mathbf{G}(k)$ operates naturally on this space. $\mathcal{S}_{c}(X)$ is usually called the space of principle series representations. One may observe, however, that for certain purposes $\mathcal{S}_{c}(X)$ is not the best possible space of functions on $X$. One way to see this is the following.

Let $\mathbf{T}$ denote the Cartan group of $\mathbf{G}$. Then $\mathbf{T}$ acts on $\mathbf{X}$ and this action commutes with the $\mathbf{G}$-action. Hence $T=\mathbf{T}(k)$ acts on $\mathcal{S}_{c}(X)$ and this action commutes with the $G$-action on $\mathcal{S}_{c}(X)$. Let $\chi$ be a character of $T$. Then we denote by $I_{\chi}$ the space of coinvariants of $T$ on $\mathcal{S}_{c}(X)$ with respect to the character $\chi$. Then $I_{\chi}$ is an admissible representation of $G$, which is irreducible for generic $\chi$ ("I" here stands for "induced", since $I_{\chi}$ is an induced representation of $G$ ). Then it is well-known that there is a natural action $\chi \mapsto w_{\bullet} \chi$ of $W$ on the set of all characters of $T$ such that $I_{\chi}$ is isomorphic $I_{w_{\bullet} \chi}$ for generic $\chi$ (cf. (2.6)

The work of both authors was partially supported by the Natural Science Foundation. 
for the definition of this action). However, this is not true for all characters $\chi$. The main purpose of this paper is "to correct" this difficulty. Namely we define certain bigger space $\mathcal{S}(X)$ of functions on $X$ (which we call the Schwartz space of $X$ ), which is $G \times T$-invariant and such that

1) $\mathcal{S}_{\chi}=I_{\chi}$ for generic $\chi$, where $\mathcal{S}_{\chi}$ denotes the space of $(T, \chi)$-coinvariants in $\mathcal{S}(X)$.

2) There is a natural action of $W$ on $\mathcal{S}(X)$, commuting with the $G$-action, which gives rise to the isomorphisms $\mathcal{S}_{\chi} \widetilde{\rightarrow} \mathcal{S}_{w_{\bullet} \chi}$ for any $w \in W$ and any character $\chi$ of $T$.

1.2. Example: $\mathbf{G}=S L(2)$. Suppose now that $\mathbf{G}=S L(2)$. In this case $\mathbf{X}=\mathbb{A}^{2} \backslash\{0\}$ (here $\mathbb{A}^{2}$ denotes the affine plane), hence $X=k^{2} \backslash\{0\}$. In this case we define $\mathcal{S}(X)$ to be space of locally constant, compactly supported functions on $k^{2}$. One can check directly that it satisfies conditions 1 and 2 above.

1.3. The general case. Unlike the case of $\mathbf{G}=S L(2)$, for general group $\mathbf{G}$ the space $\mathcal{S}(X)$ will not have any natural realization as the space $C_{c}^{\infty}(\mathbf{Y}(k))$ of locally constant compactly supported functions on $\mathbf{Y}(k)$ for an algebraic variety $\mathbf{Y}$ over $k$. So, to define it we need to adopt a rather different strategy. It was shown by Gelfand and Graev (cf. [6] and references therein) that the space $L^{2}(X)$ of $L^{2}$-functions on $X$ admits a natural action of $W$ by unitary operators $\Phi_{w}$. The operators $\Phi_{w}$ can be thought of as generalized Fourier transforms (cf. Section 2.2 for the definition of $\Phi_{w}$ ). We define $\mathcal{S}(X)$ to be equal tthe subspace of $L^{2}(X)$, equal to the sum of $\Phi_{w}\left(\mathcal{S}_{c}(X)\right)$ over all elements $w \in W$ (the sum is taken inside $\left.L^{2}(X)\right)$. Despite the fact that this definition might seem rather artificial, it turns out the the space $\mathcal{S}(X)$ has a lot of nice properties (the reader should compare our definition of $\mathcal{S}(X)$ with the definition of affine Hecke algebras given in [3]).

It would be very interesting to generalize our construction to the space of functions on $\mathbf{G} / \mathbf{U}_{\mathbf{P}}(k)$ for any parabolic subgroup $\mathbf{P}$ of $\mathbf{G}$ (here $\mathbf{U}_{\mathbf{P}}$ denotes the unipotent radical of $\mathbf{P}$ ). However, we don't know how to do this, since the definition of the operators, analogous to $\Phi_{w}$ seems to be much more complicated in the parabolic case.

1.4. This paper is organized as follows. Section 2 contains some basic notations and preliminaries about the basic affine space $\mathbf{X}$. In Section 3 we define the Schwartz space $\mathcal{S}(X)$ of functions on $X$ and describe explicitely the space $K$-ivariant vectors there, where $K$ is a maximal compact subgroup in $G \times T$. In Section 1 we study the space of $I$-invariant vectors in $\mathcal{S}(X)$, where $I \subset G$ is an Iwahori subgroup. In particular we show that this space coincides (after suitable identifications) with the space, spanned by the elements of the so called "periodic Lusztig's basis". In Section 5 we continue working with the space $\mathcal{S}(X)^{I}$ give another construction of it, using certain equivariant K-group (thus reproving a result by G. Lusztig - cf. [12]). In Section 6 we give certain convenient explicit description of the space $\mathcal{S}^{\prime}(X)$, which is the dual space to $\mathcal{S}(X)$ (this description is, in some sense, analogous to the main result in [3]). Finally in Section $\square$ we discuss an analogue of the 
space $\mathcal{S}(X)$ for a global field $F$. In particular we give a somewhat untraditional treatment of the theory of (principle) Eisenstein series, which is morally similar to the definition of geometric Eisentein series (cf. [1], [2]).

1.5. Acknowledgements. We are very grateful J. Bernstein for a lot of useful discussions on the subject and to G. Lusztig for numerous helpful conversations and remarks and for letting us know the contents of [12]. We would also like to thank V. Ginzburg for pointing out a similarity between Theorem 6.2 and the main result of [3]. The first author is also indebted to W. Soergel who first introduced him to the contents of [11].

\section{NotATIONS AND PRELIMINARIES}

2.1. Notations. Let $k$ be a local non-arcimedean field, $\mathcal{O} \subset k$-its ring of integers. We will denote algebraic varieties over $k$ by bold letters and their sets of $k$-points by the corresponding ordinary letters. Let $q$ denote the number of elements in the residue field of $k$. Let also $\pi$ be a uniformiser of $k$. We will suppose that the norm on $k$ is normalized in such a way that $\|\pi\|=q^{-1}$.

Let $\mathbf{G}$ be reductive, connected, simply connected algebraic group over $k$. We will suppose also that $\mathbf{G}$ is split over $k$ and that its derived group $[\mathbf{G}, \mathbf{G}]$ is simply connected. Let $\mathbf{U}$ be a $k$-rational maximal unipotent subgroup of $k$. Set $\mathbf{X}=\mathbf{G} / \mathbf{U}$. We will refer to $\mathbf{X}$ as the basic affine space of $\mathbf{G}$. The group $\mathbf{G}$ acts naturally on $\mathbf{X}$ (on the left).

Let $\mathbf{B}$ be the Borel subgroup of $\mathbf{G}$ which contains $\mathbf{U}$ and let $\mathbf{T}=\mathbf{B} / \mathbf{U}$ be the corresponding Cartan group. Then $\mathbf{T}$ also acts naturally on $\mathbf{X}$ (on the right) and this action commutes with the $\mathbf{G}$-action on $\mathbf{X}$.

The variety $\mathbf{X}$ is quasi-affine. Let $\overline{\mathbf{X}}$ denote its affine closure. One has a natural G-equivariant disjoint decomposition

$$
\overline{\mathbf{X}}=\bigcup \mathbf{X}_{\mathbf{P}}
$$

where the union is taken over all conjugacy classes of parabolic subgroups in $\mathbf{G}$ and $\mathbf{X}_{\mathbf{P}}=\mathbf{G} /[\mathbf{P}, \mathbf{P}]$. Set now

$$
\overline{\mathbf{X}}^{\mathrm{reg}}=\mathbf{X} \cup\left(\underset{\mathbf{P} \text { subminimal }}{\bigcup_{\mathbf{P}}} \mathbf{X}_{\mathbf{P}}\right.
$$

(by subminimal parabolic we mean a parabolic subgroup $\mathbf{P}$ whose Levi group has semisimple rank 1). The variety $\overline{\mathbf{X}}^{\text {reg }}$ is the maximal non-singular open subset of $\overline{\mathbf{X}}$.

Set $G=\mathbf{G}(k), T=\mathbf{T}(k), X=\mathbf{X}(k)$. Let $\Gamma=T / \mathbf{T}(\mathcal{O})$ be the coroot lattice of $\mathbf{G}$. We will identify every coroot $\alpha^{\vee}: \mathbb{G}_{m} \rightarrow \mathbf{T}$ with its image in $\Gamma$ (the latter is welldefined, since the group $k^{\times} / \mathcal{O}^{\times}$is canonically identified with $\mathbb{Z}$ ). We will denote by $\Delta^{\vee}$ the set of coroots of $\mathbf{G}$ and by $\Delta_{+}^{\vee}$ the set of positive coroots. We will also denote by $\Gamma^{+}$ 
the semigroup of positive elements in $\Gamma$. We also let val : $T \rightarrow \Gamma$ denote the natural projection.

Let $\Gamma^{\vee}$ denote the dual lattice to $\Gamma$. This is the lattice of weights of G. Also let $\widetilde{\Gamma}$ denote the coweight lattice of $\mathbf{G}$ (thus $\Gamma \subset \widetilde{\Gamma}$ is a subgroup of finite index). Let $\Pi \subset \Delta_{+} \subset \Delta \subset \Gamma^{\vee}$ be respectively the set of simple roots, the set of positive roots and the set of all roots of $\mathbf{G}$. Let also $\rho \in \Gamma^{\vee}$ be the half-sum of all positive roots.

Let $K=\mathbf{G}(\mathcal{O}) \times \mathbf{T}(\mathcal{O})$. Then $K$-orbits on $X$ are in natural one-to-one correspondence with elements of $\Gamma$ (in fact any $\mathbf{G}(\mathcal{O})$-orbit on $X$ is automatically a $K$-orbit, so in what follows one may use $\mathbf{G}(\mathcal{O})$ instead of $K$ ). This correspondence can be described in the following way. Let $\gamma \in \Gamma$ and let $t_{\gamma}$ be a representative of $\gamma$ in $T$. Then we set $X_{\gamma}=$ $\mathbf{G}(\mathcal{O}) t_{\gamma} \mathbf{T}(\mathcal{O}) / U$. It is easy to see that

$$
X=\bigcup_{\gamma \in \Gamma} X_{\gamma}
$$

and that this union is disjoint.

The variety $\mathbf{X}$ admits unique up to a constant top-degree $\mathbf{G}$-invariant differential form, which by [13] produces a $G$-invariant measure on $X$. It is easy to see that for such a measure one has

$$
\operatorname{vol}\left(X_{\gamma}\right)=q^{-2\langle\gamma, \rho\rangle} \operatorname{vol}\left(X_{0}\right)
$$

Therefore, we see that the sum $\sum_{\gamma \in \Gamma^{+}} \operatorname{vol}\left(X_{\gamma}\right)$ is convergent. We will normalize our measure by requiring that

$$
\operatorname{vol}\left(\bigcup_{\gamma \in \Gamma^{+}} X_{\gamma}\right)=1
$$

Let $C^{\infty}(T)$ denote the space of locally constant functions on $T$. For any such function $a$ and any $w \in W$ we set

$$
w_{\bullet}(a)(t)=q^{\langle\operatorname{val}(t), w(\rho)-\rho\rangle} a\left(w^{-1}(t)\right)
$$

This formula defines an action of $w$ on $C^{\infty}(T)$.

2.2. Fourier transforms (normalized intertwining operators). In what follows we fix a non-trivial character $\psi: F \rightarrow \mathbb{C}^{*}$.

Let $L^{2}(X)$ denote the space of $L^{2}$-functions on $X$ with respect to the above measure. By [6] this space admits a natural action of the Weyl group $W$ of $\mathbf{G}$ by means of certain unitary operators $\Phi_{w}$. Let $\alpha$ be a simple root of $\mathbf{G}$ and let $s_{\alpha} \in W$ be the corresponding simple reflection. Set $\Phi_{\alpha}=\Phi_{s_{\alpha}}$. Then the operator $\Phi_{\alpha}$ can be explicitly described as follows.

For a simple root $\alpha$ let $\mathbf{P}_{\alpha} \subset \mathbf{G}$ be the minimal parabolic of type $\alpha$ containing $B$. Let $\mathbf{B}_{\alpha}$ be the commutator subgroup of $P_{\alpha}$, and denote $\mathbf{X}_{\alpha}:=\mathbf{G} / \mathbf{B}_{\alpha}$. We have an 
obvious projection of homogeneous spaces $\pi_{\alpha}: \mathbf{X} \rightarrow \mathbf{X}_{\alpha}$. It is a fibration with the fiber $\mathbf{B}_{\alpha} / \mathbf{U}=\mathbb{A}^{2}-\{0\}$.

Let $\bar{\pi}_{\alpha}: \overline{\mathbf{X}}^{\alpha} \rightarrow \mathbf{X}_{\alpha}$ be the relative affine completion of the morphism $\pi_{\alpha}$. (So $\bar{\pi}_{\alpha}$ is the affine morphism corresponding to the sheaf of algebras $\pi_{\alpha *}\left(\mathcal{O}_{\mathbf{X}}\right)$ on $\left.\mathbf{X}_{\alpha}.\right)$ Then $\bar{\pi}_{\alpha}$ has the structure of a 2-dimensional vector bundle; $\mathbf{X}$ is identified with the complement to the zero-section in $\overline{\mathbf{X}}^{\alpha}$. The $\mathbf{G}$-action on $\mathbf{X}$ obviously extends to $\overline{\mathbf{X}}^{\alpha}$; moreover, it is easy to see that the determinant of the vector bundle $\bar{\pi}_{\alpha}$ admits a canonical (up to a constant) G-invariant trivialization, i.e. $\bar{\pi}_{\alpha}$ admits unique up to a constant G-invariant fiberwise symplectic form $\omega_{\alpha}$. We will fix such a form for every $\alpha$.

Obviously $L^{2}(X)=L^{2}\left(\bar{X}^{\alpha}\right)$. Thus we define $\Phi_{\alpha}$ to be equal to the Fourier transform in the fibers of $\bar{\pi}_{\alpha}$, corresponding to the identification of $\bar{X}^{\alpha}$ with the dual bundle by means of $\omega_{\alpha}$.

\section{The SCHWARTZ SPACE}

3.1. Definition of the Schwartz space. Let $\mathcal{S}_{c}(X)$ denote the space of smooth functions with compact support on $X$ (by a smooth function on $X$ we mean a function which is invariant under some open compact subgroup of $G \times T)$. Clearly $\mathcal{S}_{c}(X) \subset L^{2}(X)$. Recall that the Weyl group $W$ acts on $L^{2}(X)$ by means of the operators $\Phi_{w}$. These operators do not preserve the subspace $\mathcal{S}_{c}(X)$. We define now

$$
\mathcal{S}(X)=\sum_{w \in W} \Phi_{w}\left(\mathcal{S}_{c}(X)\right) ; \quad \mathcal{S}^{0}(X)=\bigcap_{w \in W} \Phi_{w}\left(\mathcal{S}_{c}(X)\right)
$$

Both the sum and the intersection are taken in $L^{2}(X)$. We have obvious inclusions

$$
\mathcal{S}^{0}(X) \subset \mathcal{S}_{c}(X) \subset \mathcal{S}(X) \subset L^{2}(X)
$$

3.2. Example. Let $\mathbf{G}=S L(2)$. In this case $X$ is isomorphic to $k^{2} \backslash\{0\}$ (here $\{0\} \in k^{2}$ denotes the origin) with the natural action of $G=S L(2, k)$ and $T=k^{\times}$on it. We claim now that $\mathcal{S}(X)$ in this case coincides with the space $C_{c}^{\infty}\left(k^{2}\right)$ of locally constant compactly supported functions on $k^{2}$.

Indeed, we have the obvious inclusion $\mathcal{S}_{c}(X) \subset C_{c}^{\infty}\left(k^{2}\right)$. On the other hand, $C_{c}^{\infty}\left(k^{2}\right)$ is invariant under the operator $\Phi_{\alpha}$ (where $\alpha$ is the unique simple root of $S L(2)$ ), since in this case $\Phi_{\alpha}$ just coincides with the symplectic Fourier transform. Hence $\mathcal{S}(X)$ is contained in $C_{c}^{\infty}\left(k^{2}\right)$.

Let us prove the opposite inclusion. Let $c_{0}$ denote the characteristic function of $\mathcal{O}^{2} \subset k^{2}$. Then it is enough to show that $c_{0} \in \mathcal{S}(X)$ since any $f \in C_{c}^{\infty}\left(k^{2}\right)$ can be decomposed as

$$
f=\left(f-f(0) c_{0}\right)+f(0) \delta_{0}
$$

and $f-f(0) c_{0} \in \mathcal{S}_{c}(X)$. 
For any $n \in \mathbb{Z}$ define functions $c_{n} \in C_{c}^{\infty}\left(k^{2}\right)$ and $\delta_{n} \in \mathcal{S}_{c}(X)$ by

$$
c_{n}(x)=\left\{\begin{array}{ll}
q^{n} & \text { if } x \in \pi^{n} \mathcal{O}^{2} \\
0 & \text { otherwise }
\end{array} \quad \delta_{n}(x)= \begin{cases}q^{n} & \text { if } x \in \pi^{n} \mathcal{O}^{2} \backslash \pi^{n+1} \mathcal{O}^{2} \\
0 & \text { otherwise }\end{cases}\right.
$$

Then $\Phi_{\alpha}\left(c_{n}\right)=c_{-n}$. Hence

$$
\begin{aligned}
& \Phi_{\alpha}\left(\delta_{n}\right)=\Phi_{\alpha}\left(c_{n}-q^{-1} c_{n+1}\right)= \\
& c_{-n}-q^{-1} c_{-n-1}=\left(1-q^{-2}\right)\left(c_{-n}-q^{-1} \delta_{-n-1}\right)
\end{aligned}
$$

Therefore $c_{0}=\left(1-q^{-2}\right)^{-1}\left(\Phi_{\alpha}\left(\delta_{0}\right)+q^{-1} \delta_{-1}\right)$ which shows that $c_{0} \in \mathcal{S}(X)$.

For general $\mathbf{G}$ we cannot give such a simple description of $\mathcal{S}(X)$. However, the following result holds.

Lemma 3.3. 1. Any function $f \in \mathcal{S}(X)$ is well-defined and locally constant on $\bar{X}^{\text {reg }}$.

2. For any $f \in \mathcal{S}(X)$ let $\operatorname{supp}_{\Gamma}(f)$ denote the set of all $\gamma \in \Gamma$ such that $\left.f\right|_{X_{\gamma}} \neq 0$. Then there exists an element $\gamma \in \Gamma$ such that $\operatorname{supp}_{\Gamma}(f) \subset \gamma+\Gamma^{+}$.

Proof. First of all, we claim that 2 implies 1 . Indeed, we must show that for any $w \in W$ and any $f \in \mathcal{S}_{c}(X)$ the function $\Phi_{w}(f)$ satisfies 1. Let us prove this by induction on $\ell(w)$. So, assume that 1 holds for some $w \in W$. Take any $\alpha \in \Pi$. We must show that $\Phi_{s_{\alpha} w}(f)=\Phi_{\alpha}\left(\Phi_{w}(f)\right)$ satisfies 1 . It is easy to see from the definition of $\Phi_{\alpha}$ that for any simple root $\alpha$ of $\mathbf{G}$ and any locally constant function $g$ on $X$, which satisfies both 1 and 2 the function $\Phi_{\alpha}(g)$ is well-defined and satisfies 1 . Taking now $g=\Phi_{w}$ we see that 2 implies 1.

So, let us prove 2. This statement can be reformulated as follows: for every $f \in \mathcal{S}_{c}(X)$ and every $w \in W$ there exists a locally constant function $a$ on $T$ such that $\Phi_{w}(f)=f^{\prime} * a$ where $f^{\prime} \in \mathcal{S}_{c}(X)$ and such that $a(t)=0$ if $\operatorname{val}(t) \notin \Gamma^{+}$. Let us prove this by induction on $\ell(w)$.

First of all, if $\ell(w)=1$, i.e. $w=s_{\alpha}$ is a simple reflection, then the statement is clear. Moreover, in this case one can choose $a$ in such a way that $a(t) \neq 0$ only if $\operatorname{val}(t)=n \alpha^{\vee}$ for $n \in \mathbb{N}$.

Assume now by induction, that we know our statement for some $w \in W$ and let $\alpha \in \Pi$ be a simple root, such that $\ell\left(w s_{\alpha}\right)=\ell(w)+1$. Let $f \in \mathcal{S}_{c}(X)$. Let $a_{1}, a_{2} \in C^{\infty}(T)$ be two functions, such that $\Phi_{\alpha}(f)=f^{\prime} * a_{1}$ and $\Phi_{w}\left(f^{\prime}\right)=f^{\prime \prime} * a_{2}$ such that all the above properties hold, i.e. $f^{\prime}, f^{\prime \prime} \in \mathcal{S}_{c}(X), a_{1}(t) \neq 0$ only if $\operatorname{val}(t)=n \alpha^{\vee}$ with $n>0$ and $a_{2}(t)=0$ if $\operatorname{val}(t) \notin \Gamma^{+}$. Then $\Phi_{w s_{\alpha}}(f)=f^{\prime \prime} *\left(a_{2} * w_{\bullet}\left(a_{1}\right)\right)$ where $w_{\bullet}\left(a_{1}\right)(t)=q^{\langle\operatorname{val}(w(t)), \rho\rangle} a_{1}(w(t))$. So, it is enough to check that $a_{2} * w_{\bullet}\left(a_{1}\right)(t)=0$, if $\operatorname{val}(t) \notin \Gamma^{+}$. By the definition

$$
a_{2} * w_{\bullet}\left(a_{1}\right)(t)=\int_{\tau \in T} q^{\langle v a l(w(\tau)), \rho\rangle} a_{2}\left(t \tau^{-1}\right) a_{1}\left(w^{-1}(\tau)\right) d \tau
$$


We claim now that if $\operatorname{val}(t) \notin \Gamma^{+}$then $a_{2}\left(t \tau^{-1}\right) a_{1}\left(w^{-1}(\tau)\right)=0$ for any $\tau \in T$. Indeed, if $\operatorname{val}\left(w^{-1}(\tau)\right) \neq n \alpha^{\vee}$ for some $n \in \mathbb{N}$, then $a_{1}\left(w^{-1}(\tau)\right)=0$. So, suppose that $\operatorname{val}\left(w^{-1}(\tau)\right)=$ $n \alpha^{\vee}$ with $n \in \mathbb{N}$, i.e. $\operatorname{val}(\tau)=n w\left(\alpha^{\vee}\right)$. But the condition $\ell\left(w s_{\alpha}\right)=\ell(w)+1$ implies that $w\left(\alpha^{\vee}\right) \in \Gamma^{+}$. Hence $\operatorname{val}(\tau)>0$. Therefore $\operatorname{val}\left(t \tau^{-1}\right) \notin \Gamma^{+}$which by our assumptions implies that $a_{2}\left(t \tau^{-1}\right)=0$. This finishes the proof.

3.4. The algebra $\mathcal{A}$. In what follows we fix a Haar measure on $T$. We willnormalize it by requiring that the volume of $\mathbf{T}(\mathcal{O})$ is equal to 1 . Let $\mathcal{A}$ denote the space of locally compact, compactly supported functions on $T$. Then $\mathcal{A}$ has a natural structure of a commutative algebra with respect to convolution.

Let $\chi$ be a character of $\mathbf{T}(\mathcal{O})$. Denote by $\mathcal{A}_{\chi}$ the subspace of $\mathcal{A}$, consisting of all functions $f \in \mathcal{A}$ such that $f(t x)=\chi\left(t^{-1}\right) f(x)$ for any $t \in \mathbf{T}(\mathcal{O}), x \in T$. Then $\mathcal{A}_{\chi}$ is a subalgebra of $\mathcal{A}$, which is in fact isomorphic to $\mathbb{C}[\Gamma]=\mathcal{A}_{0}$ for any $\chi$. Moreover, one has a direct sum decomposition $\mathcal{A}=\oplus \mathcal{A}_{\chi}$. Let also $Z_{\chi}=\operatorname{Spec} \mathcal{A}_{\chi}$.

The algebra $\mathcal{A}$ acts naturally on any smooth representation of $T$ and, in particular, on the spaces $\mathcal{S}^{0}(X), \mathcal{S}_{c}(X)$ and $\mathcal{S}(X)$. If $V$ is any smooth representation of $T$ we set

$$
V^{\chi}=\{v \in V \mid t(v)=\chi(t) v \text { for any } t \in \mathbf{T}(\mathcal{O})\}
$$

The space $V^{\chi}$ has a natural structure of an $\mathcal{A}_{\chi}$-module.

Let $\pi: V_{1} \rightarrow V_{2}$ be a morphism of smooth $T$-modules.

Definition 3.5. We say that $\pi$ is an isomorphism at the generic point of $\mathcal{A}$ if for every character $\chi$ of $\mathbf{T}(\mathcal{O})$ the induced morphism $\pi_{\chi}: V_{1}^{\chi} \rightarrow V_{2}^{\chi}$ of $\mathcal{A}_{\chi}$-modules is an isomorphism at the generic point of $Z_{\chi}$.

Lemma 3.6. The embeddings $\mathcal{S}^{0}(X) \hookrightarrow \mathcal{S}_{c}(X) \hookrightarrow \mathcal{S}(X)$ are isomorphisms at the generic point of $\mathcal{A}$.

Proof. First of all, it is easy to see that the fact that the map $\mathcal{S}^{0}(X) \hookrightarrow \mathcal{S}_{c}(X)$ is an isomorphism at the generic point of $\mathcal{A}$ implies that the map $\mathcal{S}_{c}(X) \hookrightarrow \mathcal{S}(X)$ is an isomorphism at the generic point of $\mathcal{A}$.

So, let us show that the map $\mathcal{S}^{0}(X) \hookrightarrow \mathcal{S}_{c}(X)$ is an isomorphism at the generic point of $\mathcal{A}$. Let $\rho$ be a character of $T$. Then $\rho$ defines a point in $Z_{\chi}$ where $\chi=\left.\rho\right|_{\mathbf{T}(\mathcal{O})}$. Let $I_{\rho}=\operatorname{Ind}_{B}^{G}(\rho)$ denote the corresponding induced representation (here we regard $\rho$ as a character of $B$ by means of the identification $B / U=T)$. Let also

$$
\begin{aligned}
I_{\rho}^{0}= & \mathcal{S}^{0}(X) / \operatorname{span}\left\{f \in \mathcal{S}^{0}(X) \mid f=t^{*}(g)-\rho(t)^{-1} g\right. \\
& \text { for some } \left.t \in T, g \in \mathcal{S}^{0}(X)\right\}
\end{aligned}
$$

Then we have the natural map $p_{\rho}: I_{\rho}^{0} \rightarrow I_{\rho}$. 
Now in order to show the the embedding $\mathcal{S}^{0}(X) \hookrightarrow \mathcal{S}_{c}(X)$ is an isomorphism at the generic point of $\mathcal{A}$ it is enough to show that $p_{\rho}$ is surjective for generic $\rho$. However, it is well known that for generic $\rho$ the $G$-module $I_{\rho}$ is irreducible. Hence we just have to show that for generic $\rho$ the morphism $p_{\rho}$ is non-zero.

Recall that a character $\Psi$ of $U$ is called non-degenerate if for any $\alpha \in \Pi$, the restriction of $\Psi$ to the one-parametric subgroup $U_{\alpha}$, corresponding to $\alpha$, is non-trivial. Let now $\Psi$ be a non-degenerate character of $U$. Set $W_{\Psi}=\operatorname{Ind}_{U}^{G}(\Psi)$ to be the corresponding induced representation. Then it is well-known that for generic $\rho$ one has $\operatorname{Hom}_{G}\left(I_{\rho}, W_{\Psi}\right) \neq 0$. On the other hand by [6] we have

$$
\operatorname{Hom}_{G}\left(\mathcal{S}_{c}(X), W_{\Psi}\right) \simeq \operatorname{Hom}_{G}\left(\mathcal{S}^{0}(X), W_{\Psi}\right)
$$

where the isomorphism is induced by the embedding $\mathcal{S}^{0}(X) \hookrightarrow \mathcal{S}_{c}(X)$. This implies that $p_{\rho} \neq 0$ for generic $\rho$.

3.7. Some pairings. Let $A$ be a commutative algebra and let $M$ be a module over $A$. Then $M^{\vee}=\operatorname{Hom}_{A}(M, A)$ also has a natural structure of an $A$-module. Moreover we have a natural map $M \rightarrow\left(M^{\vee}\right)^{\vee}$. Recall that an $A$-module $M$ is called reflexive if the above map is an isomorphism. Clearly any finitely generated free $A$-module is reflexive.

Let $V$ be any representation of $G \times T$. We will say that it is $\mathcal{A}$-reflexive if for any open compact subgroup $H$ of $G$ and any character $\chi$ of $\mathbf{T}(\mathcal{O})$ the space $V^{H, \chi}$ is reflexive as an $\mathcal{A}_{\chi}$-module (here $V^{H, \chi}$ denotes the space of all elements of $V$, which are invariant with respect to $H$ and on which $\mathbf{T}(\mathcal{O})$ acts by means of $\chi)$.

For any smooth $G \times T$-module $V$ we define

$$
V^{\vee}=\sum_{\chi} \bigcup_{H} \operatorname{Hom}_{\mathcal{A}_{\chi}}\left(V^{H, \chi}, \mathcal{A}_{\chi}\right)
$$

Note that $V^{\vee}$ also have a structure of a $G \times T$-module. It is easy to see that if we have an isomorphism $V \simeq V_{1}^{\vee}$ for some $G \times T$-module $V_{1}$, which is isomorphic at the genric point of $\mathcal{A}$ to an $\mathcal{A}$-reflexive module, then $V$ is also $\mathcal{A}$-reflexive.

Theorem 3.8. The $G \times T$-modules $\mathcal{S}^{0}(X)$ and $\mathcal{S}_{c}(X)$ are $\mathcal{A}$-reflexive. Moreover, one has natural identifications

$$
\mathcal{S}(X)^{\vee} \simeq \mathcal{S}^{0}(X) \text { and } \mathcal{S}_{c}(X)^{\vee} \simeq \mathcal{S}_{c}(X)
$$

These isomorphisms are compatible with the embeddings $\mathcal{S}^{0}(X) \subset \mathcal{S}_{c}(X)$ and $\mathcal{S}_{c}(X) \subset$ $\mathcal{S}(X)$. Moreover, the first isomorphism in (3.12) is $W$-equivariant (recall, that $W$ acts both on $\mathcal{S}^{0}(X)$ and on $\mathcal{S}(X)$ by $\left.\Phi_{w}\right)$.

Proof. The fact that $\mathcal{S}_{c}(X)$ is $\mathcal{A}$-reflexive follows easily from the fact that $T$ acts freely on $X$ and from the fact that for any compact open subgroup $H$ of $G$ the set of $H \times T$-orbits on $X$ is finite. 
Define now a pairing $\langle\cdot, \cdot\rangle: \mathcal{S}_{c}(X) \otimes \mathcal{S}_{c}(X) \rightarrow \mathcal{A}$ by

$$
\langle f, g\rangle(t)=q^{-\langle\operatorname{val}(t), \rho\rangle}\left(t^{*} f, g\right)_{L^{2}}
$$

Here $(\cdot, \cdot)_{L^{2}}$ denotes the $L^{2}$ scalar product and $t^{*} f(x)=f(t x)$.

It is easy to see that this pairing is $\mathcal{A}$-equivariant and that it defines an isomorphism between $\mathcal{S}_{c}(X)$ and $\mathcal{S}_{c}(X)^{\vee}$.

Note now that the expression in the right hand side of (3.10) makes sense for any two smooth functions $f$ and $g$ if we suppose that $g \in \mathcal{S}_{c}(X)$. However, if we don't suppose that $f \in \mathcal{S}_{c}(X)$, it might happen that the resulting function $\langle f, g\rangle$ does not have compact support and, therefore, does not lie in $\mathcal{A}$.

Lemma 3.9. $\quad$ 1. Suppose that we are given $f \in \mathcal{S}(X), g \in \mathcal{S}^{0}(X)$. Then the function $\langle f, g\rangle$ defined by (3.10) lies in $\mathcal{A}$ and therefore (3.10) defines an $\mathcal{A}$-equivariant pairing $\langle\cdot, \cdot\rangle: \mathcal{S}(X) \otimes \mathcal{S}^{0}(X) \rightarrow \mathcal{A}$.

2. The above pairing is $W$-equivariant.

3. The above pairing gives rise to an isomorphism $\mathcal{S}^{0}(X) \simeq \mathcal{S}(X)^{\vee}$.

4. The space $\mathcal{S}^{0}(X)$ is $\mathcal{A}$-reflexive.

Proof. To prove 1 we must show that $\langle f, g\rangle$ has compact support. For this it enough to show that the set of all $\gamma \in \Gamma$ such that $\langle f, g\rangle_{\gamma \mathbf{T}(\mathcal{O})} \neq 0$ is finite. Let us denote this set by $Z_{f, g}$. Then it follows from Lemma 3.3(2) that there exists $\gamma \in \Gamma$ such that $Z_{f, g}$ is contained in $\gamma+\Gamma^{+}$. On the other hand, the same is true also for the set $Z_{\Phi_{w}(f), \Phi_{w}(g)}$. But $Z_{\Phi_{w}(f), \Phi_{w}(g)}=w\left(Z_{f, g}\right)$, which implies that $Z_{f, g}$ is finite.

The second statement in Lemma 3.9 is obvious. Let us prove the third one. First of all let us show that our pairing defines an isomorphism $\mathcal{S}^{0}(X) \simeq \mathcal{S}(X)^{\vee}$. Indeed, we have a map $\mathcal{S}^{0}(X) \rightarrow \mathcal{S}(X)^{\vee}$. It is easy to see that this map is injective. On the other hand, it also easy to see that we have a natural embedding $\mathcal{S}(X)^{\vee} \rightarrow \mathcal{S}_{c}(X)$ (this follows for example from Lemma 3.6). So, let $g \in \mathcal{S}(X)^{\vee} \subset \mathcal{S}_{c}(X)$. Then, since $\mathcal{S}(X)$ is invariant under the operators $\Phi_{w}$, it follows that $\Phi_{w}(g) \subset \mathcal{S}(X)^{\vee}$ for any $w \in W$. Hence $\Phi_{w}(g) \in \mathcal{S}_{c}(X)$ for any $w \in W$ which implies that $g \in \mathcal{S}^{0}(X)$. Hence $\mathcal{S}^{0}(X)=\mathcal{S}(X)^{\vee}$ which implies also that $\mathcal{S}^{0}(X)$ is $\mathcal{A}$-reflexive.

3.10. A generalization. Let $Z \subset W$ be any subset. Then we can define spaces $\mathcal{S}^{Z}(X)$ and $\mathcal{S}_{0}^{Z}(X)$ by

$$
\mathcal{S}^{Z}(X)=\sum_{w \in Z} \Phi_{w}\left(\mathcal{S}_{c}(X)\right) ; \quad \mathcal{S}_{0}^{Z}(X)=\bigcap_{w \in Z} \Phi_{w}\left(\mathcal{S}_{c}(X)\right)
$$


Then Theorem 3.8 can be generalized in the following way (we will need this generalization in Section (6).

Theorem 3.11. The $G \times T$-modules $\mathcal{S}_{0}^{Z}(X)$ is $\mathcal{A}$-reflexive. Moreover, one has a natural identification

$$
\mathcal{S}_{0}^{Z}(X) \simeq \mathcal{S}^{Z}(X)^{\vee}
$$

The proof of this result is the same as the proof of Theorem 3.8 and it is left to the reader.

3.12. Description of $K$-invariant vectors. Set now $K=\mathbf{G}(\mathcal{O}) \times \mathbf{T}(\mathcal{O})$. Thus $K$ is a maximal compact subgroup of $G \times T$. In this subsection we will give an explicit description of $K$-invariant elements of $\mathcal{S}(X)$ (in fact, as it is observed in Section 2, one has $\mathcal{S}(X)^{K}=\mathcal{S}(X)^{\mathbf{G}(\mathcal{O})}$. So, in what follows, one may replace $K$ by $\mathbf{G}(\mathcal{O})$ ).

3.12.1. The functions $\delta_{\gamma}$. Recall that $\Gamma$ denotes the coroot lattice of $\mathbf{G}$ and that one has a disjoint decomposition

$$
X=\bigcup_{\gamma \in \Gamma} X_{\gamma}
$$

Define now a function $\delta_{\gamma} \in \mathcal{S}_{c}(X)$ by

$$
\delta_{\gamma}(x)=\left\{\begin{array}{l}
q^{\langle\gamma, \rho\rangle} \quad \text { if } x \in X_{\gamma} \\
0 \quad \text { otherwise }
\end{array}\right.
$$

It is clear that the functions $\delta_{\gamma}$ form a basis in $\mathcal{S}_{c}(X)^{K}$.

3.12.2. The q-analog of Kostant's function. Let $\Gamma^{+} \subset \Gamma$ denote the semigroup of positive elements in $\Gamma$. Following Lusztig we define a function $\mathcal{K}: \Gamma^{+} \rightarrow \mathbb{C}$ by

$$
\mathcal{K}(\gamma)=\sum_{P \in \mathcal{P}_{\gamma}} q^{-|P|}
$$

where $\mathcal{P}_{\gamma}$ is the set of all representations of $\gamma$ as a sum of positive coroots and if $P \in \mathcal{P}_{\gamma}$ then we denote by $|P|$ the number of summands in $P$.

The function $\mathcal{K}(\gamma)$ may be viewed as a $q$-analog of Kostant's partition function.

3.12.3. The functions $c_{\mu}$. Let us now define a function $c_{\mu}$ on $X$ by putting

$$
c_{\mu}=\sum_{\gamma \in \Gamma^{+}} \mathcal{K}(\gamma) \delta_{\mu+\gamma}
$$

Remark. The reader should compare our definition of the function $c_{\mu}$ with the computation of stalks of intersection cohomology sheaves on Drinfeld spaces, given in [4]. 
Theorem 3.13. 1. $c_{\mu} \in \mathcal{S}(X)$ for any $\mu \in \Gamma$

2. The functions $c_{\mu}$ form a basis in $\mathcal{S}(X)^{K}$

3. $\Phi_{w}\left(c_{\mu}\right)=c_{w(\mu)}$ for any $w \in W, \mu \in \Gamma$

Proof. Let us first prove 3. First of all we claim that $e_{\mu} \in L^{2}(X)$ for any $\mu \in \Gamma$. Indeed, one has

$$
\left\|c_{\mu}\right\|_{L^{2}}^{2}=\operatorname{vol}\left(X_{0}\right)^{2} \sum_{\gamma \in \Gamma^{+}} \mathcal{K}(\gamma)^{2}
$$

So, we have to show that the sum in the right hand side of (3.16) converges. But it is easy to see that

$$
\sum_{\gamma \in \Gamma^{+}} \mathcal{K}(\gamma)=\frac{1}{\left(1-q^{-1}\right)^{\left|\Delta_{+}^{\vee}\right|}}
$$

Hence $\sum_{\gamma \in \Gamma^{+}} \mathcal{K}(\gamma)$ is convergent and therefore $\sum_{\gamma \in \Gamma^{+}} \mathcal{K}(\gamma)^{2}$ is also convergent.

Thus the expression $\Phi_{w}\left(c_{\mu}\right)$ is well defined. Clearly in order to prove Theorem 3.13 one may assume without loss of generality that $w=s_{\alpha}$ for some $\alpha \in \Pi$ and $\mu=0$.

Let us introduce some notations. Let $\gamma \in \Gamma$. Then we can define two operators $\widetilde{H}_{\gamma}$ and $H_{\gamma}$ on $\mathbf{T}(\mathcal{O})$-invariant functions on $X: \widetilde{H}_{\gamma}$ is just the operator of shift by $\gamma$ and $H_{\gamma}=q^{\langle\gamma, \rho\rangle} \widetilde{H}_{\gamma}$. Note that $\widetilde{H}_{\gamma_{1}} \widetilde{H}_{\gamma_{2}}=\widetilde{H}_{\gamma_{1}+\gamma_{2}}$ and $H_{\gamma_{1}} H_{\gamma_{2}}=H_{\gamma_{1}+\gamma_{2}}$.

The following lemma is proved by a direct calculation (which reduces essentially to $G=S L(2)$.

\section{Lemma 3.14.}

$$
\Phi_{\alpha}\left(\delta_{0}\right)=\frac{1-q^{-1} H_{-\alpha^{\vee}}}{1-q^{-1} H_{\alpha}}\left(\delta_{0}\right)
$$

Here $\frac{1}{1-q^{-1} H_{\alpha} \vee}$ is understood as a formal sum

$$
\frac{1}{1-q^{-1} H_{\alpha \vee}}=\sum q^{-n} H_{n \alpha \vee}
$$

On the other hand, we claim that the following equality holds

$$
\left(\prod_{\alpha^{\vee} \in \Delta_{+}^{\vee}} q-H_{\alpha^{\vee}}\right)\left(c_{\mu}\right)=q^{-\left|\Delta_{+}^{\vee}\right|} \delta_{\mu}
$$

where $H_{\alpha}$ denotes the operator of shift by $\alpha$. To see that (3.20) holds it is enough to note the following. Let $\widehat{\mathbb{C}}[\Gamma]$ denote the completion of $\mathbb{C}[\Gamma]$ consisting of all infinite sums $\sum a_{\gamma} H_{\gamma}$ satisfying the following condition:

- for every $\mu \in \Gamma$ the set

$$
\left\{\gamma \in \Gamma \mid a_{\gamma} \neq 0 \text { and } \gamma \notin \mu+\Gamma^{+}\right\}
$$


is finite.

It is obvious now that in $\widehat{\mathbb{C}}[\Gamma]$ we have the following equality:

$$
\frac{1}{\prod_{\alpha^{\vee} \in \Delta_{+}^{\vee}} q-H_{\alpha^{\vee}}}=q^{\left|\Delta_{+}^{\vee}\right|} \sum_{\gamma \in \Gamma^{+}} \mathcal{K}(\gamma) H_{\gamma}
$$

On the other hand (3.22) implies (3.20) because of the fact that $H_{\gamma}\left(\delta_{\mu}\right)=\delta_{\mu+\gamma}$. But (3.18) and (3.20) imply together that $\Phi_{\alpha}\left(c_{0}\right)=c_{0}$ which is precisely what we need to prove.

Let us prove 1 and 2 now. Let $N$ denote the linear span of the functions $c_{\mu}$. It is clear that $N$ is invariant under the action of $\Gamma$ (by shifts). Also Theorem 3.13(3) implies that $N$ is also invariant under $W$.

We claim now that for any $\mu \in \Gamma$ one has $\delta_{\mu} \in N$. Indeed, after one expands (3.20), one gets an expression of $\delta_{\mu}$ as a finite sum of $c_{\mu^{\prime}}$ 's with some coefficients, since $H_{\gamma}\left(c_{\mu}\right)=c_{\mu+\gamma}$.

Let $N_{c}=\mathcal{S}_{c}(X)^{K}$. It follows from the above that $N_{c} \subset N$. Hence, in order to show that $N=\mathcal{S}(X)^{K}$ (which is precisely what we have to prove), it is enough to show that the spaces $\Phi_{w}\left(N_{c}\right)$ generate $N$.

Let $\mathbf{T}^{\vee}$ denote the dual torus of $\mathbf{T}$. By definition, this is an algebraic torus over $\mathbb{C}$, whose character group is $\Gamma$. The group $W$ acts naturally on $\mathbf{T}^{\vee}$. Let $\mathbb{C}\left(\mathbf{T}^{\vee}\right)$ denote the space of regular functions on $\mathbf{T}^{\vee}$. It can be naturally identified with the group ring of $\mathbb{C}[\Gamma]$ of $\Gamma$.

It follows from Theorem 3.13(3) that the assignment $c_{\mu} \mapsto \mu$ extends to an isomorphism $N \simeq \mathbb{C}\left(\mathbf{T}^{\vee}\right)$ of $\Gamma \rtimes W$-modules, where we let $\Gamma$ act on $N$ by means of the operators $H_{\gamma}$. Moreover, it follows from (3.20) that under this isomorphism the space $N_{c}$ gets identified with the ideal of $\mathbb{C}\left(\mathbf{T}^{\vee}\right)$, generated by the function $\mathbf{d}_{q}=\prod_{\alpha \in \Delta_{+}^{\vee}}\left(q-\alpha^{\vee}\right)$ (here we consider $\alpha^{\vee}$ as a function of $\mathbf{T}^{\vee}$ with values in $\left.\mathbb{G}_{m}\right)$.

Let $\mathbf{V} \subset \mathbf{T}^{\vee}$ denote hypersurface in $\mathbf{T}^{\vee}$ defined by the equation $\mathbf{d}_{q}=0$.

Lemma 3.15. One has

$$
\bigcap_{w \in W} w(\mathbf{V})=\emptyset
$$

Proof. By Hilbert Nullstellensatz it is enough to prove that for any $\mathbf{t}^{\vee} \in \mathbf{T}^{\vee}$ there exists $w \in W$ such that $f\left(w\left(\mathbf{t}^{\vee}\right)\right) \neq 0$. However, for every $\mathbf{t}^{\vee} \in \mathbf{T}^{\vee}$ there exists $w \in W$ such that

$$
\left|\gamma\left(w\left(\mathbf{t}^{\vee}\right)\right)\right| \leq 1
$$

for every $\gamma \in \Gamma^{+}$(this means that $w\left(\mathbf{t}^{\vee}\right)$ lies in the "antidominant chamber"), which proves the lemma. 
On the other hand, it follows immediately from (3.23) that the sum all $w$-translates of the ideal, generated by $\mathbf{d}_{q}$ in $\mathbb{C}\left(\mathbf{T}^{\vee}\right)$ is equal to $\mathbb{C}\left(\mathbf{T}^{\vee}\right)$, which finishes the proof.

\section{Relation With Generic Kazhdan-Lusztig Polynomials}

In Section 4 and Section 5 we suppose that $\mathbf{G}$ is semisimple.

4.1. The affine Hecke algebra. Let $\widetilde{W}=\Gamma \rtimes W$. This is a Coxeter group. We will denote by $\widetilde{\Pi}$ the corresponding set of generators. By definition this is an algebra over the ring $\mathbb{C}\left[v, v^{-1}\right]$ of Laurent polynomials in an indeterminate $v$ with generators $T_{w}$ for $w \in \widetilde{W}$. We will denote $T_{s_{\alpha}}$ simply by $T_{\alpha}$. These generators satisfy the following relations:

$$
\begin{aligned}
& \left(T_{\alpha}+v^{-1}\right)\left(T_{\alpha}-v\right)=0 \\
& T_{w w^{\prime}}=T_{w} T_{w^{\prime}} \quad \text { if } \ell\left(w w^{\prime}\right)=\ell(w)+\ell\left(w^{\prime}\right)
\end{aligned}
$$

(here $\ell: \widetilde{W} \rightarrow \mathbb{Z}^{+}$is the length function). We will write $\mathcal{H}_{q}$ for the specialization of $\mathcal{H}$ at $v=q^{\frac{1}{2}}$. Let also $\mathcal{H}^{f} \subset \mathcal{H}$ (resp. $\mathcal{H}_{q}^{f} \subset \mathcal{H}_{q}$ ) denote the finite Hecke algebra, i.e. the subalgebra of $\mathcal{H}$ spanned by the $T_{w}$ for $w \in W$.

Let $I \subset G$ denote the Iwahori subgroup. It is well-known that $\mathcal{H}_{q}$ is isomorphic to the algebra of smooth compactly supported functions on $G$ which are $I$-bi-equivariant. This isomorphism can be characterized as follows. It is well known that the set $I \backslash G / I$ is isomorphic to $\widetilde{W}$. For any $w \in \widetilde{W}$ let $\chi_{w}$ denote the characterictic function of the corresponding double coset. Then under the above isomorphism the element $(-1)^{\ell(w)} T_{w^{-1}}^{-1}$ goes to $q^{-\frac{1}{2} \ell(w)} \chi_{w}$.

Therefore, it follows that if $V$ is any smooth representation of $G$ then the algebra $\mathcal{H}_{q}$ acts naturally on $V^{I}$.

4.2. $\mathcal{S}_{c}(X)^{I \times \mathbf{T}(\mathcal{O})}$ and the periodic Hecke module. Let Alc denote the collection of all alcoves for the group $\widetilde{W}$ in the space $\mathfrak{t}_{\mathbb{R}}$-the real Lie algebra of $\mathbf{T}$. The set Alc admits two commuting (resp. left and right actions) of the group $\widetilde{W}$. Let also $\mathcal{C}^{+} \in \mathfrak{t}_{\mathbb{R}}$ denote the dominant Weyl chamber. For any $s \in \widetilde{S}$ we will denote by $H_{s}$ the corresponding affine hyperplane in $\mathfrak{t}_{\mathbb{R}}$. For any $s \in \widetilde{S}$ we denote by $H_{s}^{+}$(resp. $H_{s}^{-}$the set of all points $x \in \mathfrak{t}_{\mathbb{R}}$ which are on the same side of $H_{s}$ as the set $\gamma+\mathcal{C}^{+}$(resp. $\gamma-\mathcal{C}^{+}$for some $\gamma \in \Gamma$. We also denote by $d:$ Alc $\rightarrow \mathbb{Z}$ the corresponding length function (cf. [10](Section 1.4)).

The set Alc has two natural (respectively left and right) actions of $\widetilde{W}$ which commute. We will denote them by $A \mapsto w A$ (resp. $A \mapsto A w$ ).

Following Lusztig we define for any $A \in$ Alc a subset $\mathcal{L}(A) \subset \widetilde{\Pi}$ in the following way. We say that $\alpha \in \mathcal{L}(A)$ if and only if the two conditions below are satisfied:

- $A \subset E_{\alpha}^{+}$.

- $A s_{\alpha} \subset E_{\alpha}^{-}$(here $A s$ denotes the right action of $s_{\alpha}$ on $A$ ). 
In [10] and [11] G. Lusztig considered the periodic Hecke module $M_{c}$ over $\mathcal{H}$. By definition this module is spanned by elements $\{A \in \mathbf{A l c}\}$ over the ring $\mathbb{C}\left[v, v^{-1}\right]$ and the action of $H$ is defined in the following way:

$$
T_{\alpha} A=\left\{\begin{array}{l}
s_{\alpha} A \quad \text { if } s \notin \mathcal{L}(A) \\
s_{\alpha} A+\left(v-v^{-1}\right) A \quad \text { if } s \in \mathcal{L}(A)
\end{array}\right.
$$

(thus, if we specialize $v$ to 1 we obtain the natural $\mathbb{C}[\widetilde{W}]$-action on $\operatorname{span}(A \mid A \in \mathbf{A l c})$ ).

The module $M_{c}$ also admits a natural action of the group $\Gamma$, which commutes with the $\mathcal{H}$-action. This action is defined by $\gamma(A)=A+\gamma$.

Let also $M_{\geq}$denote a completion of $M_{c}$, consisting of all (possibly infinite) sums $\sum m_{A} A$ such that the following property holds:

- there exists $\gamma \in \Gamma$, such that $m_{A} \neq 0$ implies that $A \subset \gamma+\mathcal{C}^{+}$.

In [11] G. Lusztig has defined for any $\alpha \in \Pi$ certain $\mathcal{H}$-linear operator $\theta_{\alpha}: M_{c} \rightarrow M_{\geq}$ in the following way. Let $A \in$ Alc. Define a sequence $A^{n}, n \geq 0$ by the consitions that $A^{n}$ lie in the same " $\alpha$-strip" as $A$ and

- $A^{0}=A s_{\alpha}$

- $d_{\alpha}\left(A^{n}, A^{n+1}\right)=1$, where $d_{\alpha}$ denotes the corresponding "partial" distance function (cf. [11]). Then

$$
\theta_{\alpha}(A)=v^{-1} A^{0}+\sum_{n=1}^{\infty}\left(v^{-n+1}-v^{-n-1}\right) A^{n}
$$

It clear, that when we specialize $v$ to 1 the operator $\theta_{\alpha}$ reduces just to an obvious operator on $M$, which comes from the right action of $W$ on Alc.

The set Alc is also in natural one-to-one correspondence with the set of $I \times \mathbf{T}(\mathcal{O})$-orbits on $X$ (in fact, as before, any $I$-invariant set is automatically $\mathbf{T}(\mathcal{O})$-invariant). So, we may write

$$
X=\bigcup_{A \in \text { Alc }} X_{A}
$$

where $X_{A}$ is the corresponding orbit. For any $A \in$ Alc we define a function $\delta_{A} \in$ $\mathcal{S}_{c}(X)^{I \times \mathbf{T}(\mathcal{O})}$ by

$$
\delta_{A}(x)=\left\{\begin{array}{l}
\left(-q^{1 / 2}\right)^{d(A)} \quad \text { if } x \in X_{A} \\
0 \quad \text { otherwise }
\end{array}\right.
$$

The following lemma can be proved by a direct computation and it is left to the reader.

Lemma 4.3. Let $M_{c, q}$ denote the specialization of $M_{c}$ at $v=q^{-\frac{1}{2}}$. Then the assignment $A \mapsto \delta_{A}$ extends to an isomorphism $\rho: M_{c, q} \underset{\rightarrow}{\rightarrow} \mathcal{S}_{c}(X)^{I \times \mathbf{T}(\mathcal{O})}$ of $\mathcal{H}_{q} \otimes \mathbb{C}[\Gamma]$-modules. Moreover, $\rho \circ \theta_{\alpha} \circ \rho^{-1}=\Phi_{\alpha}$ for any $\alpha \in \Pi$. 
4.4. In [11](Theorem 12.2) G. Lusztig has constructed certain remarkable elements $A^{\sharp} \in$ $M_{\geq}$. These elements satisfy the following conditions: first of all, $A^{\sharp}=A+v^{-1} \sum_{B} \mathbb{Z}\left[v^{-1}\right] B$. Also $A^{\sharp}$ is "self-dual" in a certain sense (cf. [11]).

Let $M_{d}=\operatorname{span}\left(A^{\sharp} A \in \mathbf{A l c}\right)$. Then it follows from Theorem 12.2 in 11 that the operators $\theta_{\alpha}$ are well-defined on $M_{d}$ and together define an action of the group $W$ on $M_{d}$. We will denote the corresponding automorphisms of $M_{d}$ by $\theta_{w}$.

Proposition 4.5. One has

$$
\sum_{w \in W} \theta_{w}\left(M_{c}\right)=M_{d}
$$

Proof. Let

$$
\mathbf{d}_{v}=\prod_{\alpha^{\vee} \in \Delta_{+}^{\vee}}\left(v^{2}-\alpha^{\vee}\right) \in \mathcal{R}_{T \times \mathbb{C}^{*}}
$$

Then it follows from [5] (Section 3) (cf. also Conjecture 13.11 in [11] and the remark afterwards) that $\mathbf{d}_{v} M_{d} \subset M_{c}$. Hence (4.7) follows from the fact the ideal generated by the elements $\left\{w\left(\mathbf{d}_{v}\right)\right\}_{w \in W}$ in $\mathcal{R}_{\mathbf{T}^{\vee} \times \mathbb{C}^{*}}$ contains 1 , which is proved exactly in the same way as Lemma 3.15

Corollary 4.6. Let $M_{d, q}$ denote the specialization of $M_{d}$ at $v=q^{\frac{1}{2}}$. Then there is an isomorphism of $\mathcal{H}_{q} \otimes \mathbb{C}[\Gamma \rtimes W]$-modules

$$
M_{d, q}=\mathcal{S}(X)^{I \times \mathbf{T}(\mathcal{O})}
$$

This follows immediately from Proposition 4.5 and Lemma 4.3.

\section{Relation With EQUivariant $K$-THEORY.}

5.1. The results of this section were also obtained earlier by G. Lusztig, using slightly different methods (cf. [12]).

5.2. Let $\mathbf{G}^{\vee}$ the Langlands dual group to $\mathbf{G}$ and let $\mathbf{T}^{\vee} \subset \mathbf{G}^{\vee}$ be its Cartan subgroup. Let also $\mathcal{B}$ denote the flag variety of $\mathbf{G}^{\vee}$. One has a natural identification $\Gamma \simeq \operatorname{Hom}\left(\mathbf{T}^{\vee}, \mathbb{C}^{*}\right)$

Let $\mathcal{K}=K_{0}^{\mathbf{T}^{\vee} \times \mathbb{C}^{*}}(\mathcal{B})$ denote the equivariant $K$-group of $\mathcal{B}$ with respect to the $\mathbf{T}^{\vee} \times \mathbb{C}^{*}$ (where $\mathbb{C}^{*}$ acts trivially on $\mathcal{B}$ ). This is a module over the ring $\mathbb{C}\left[v, v^{-1}\right]$ which can be identified with the ring of characters of $\mathbb{C}^{*}$. We will write $\mathcal{K}_{q}$ for the specialization of $\mathcal{K}$ at $v=q$.

By [7] the algebra $\mathcal{H}$ acts naturally on $\mathcal{K}$ (as in [11] we twist the action defined in [7] by means of the automorphism $\bullet: \mathcal{H} \rightarrow \mathcal{H}$ sending $T_{w}$ to $\left.(-1)^{\ell(w)} T_{w^{-1}}^{-1}\right)$. 
On the other hand, the space $\mathcal{K}$ admits also a natural action of the group $\Gamma \rtimes W$, which commutes with the action of $\mathcal{H}$. This action may be described as follows.

First of all, since $\Gamma$ is identified with the lattice of characters of $\mathbf{T}^{\vee}$, it follows that $\Gamma \simeq K_{0}^{\mathbf{T}^{\vee}}(p t)$, and therefore, $\Gamma$ acts on $K_{0}^{\mathbf{T}^{\vee}}(Y)$ for any $\mathbf{T}^{\vee}$-variety $Y$.

Suppose now that $Y$ is actually a $\mathbf{G}^{\vee}$-variety. Then the Weyl group $W$ acts naturally on $K_{0}^{\mathbf{T}^{\vee}}(Y)$ in the following way. Let $N\left(\mathbf{T}^{\vee}\right)$ denote the normalizer of $\mathbf{T}^{\vee}$ in $\mathbf{G}^{\vee}$. Then $W$ can be identified with $N\left(\mathbf{T}^{\vee}\right) / \mathbf{T}^{\vee}$. Let $w \in W$ and let $\bar{w}$ be a representative of $w$ in $N\left(\mathbf{T}^{\vee}\right)$. Let also $\mathcal{F}$ be $\mathbf{T}^{\vee}$-equivariant coherent sheaf on $Y$. Then $\left(\bar{w}^{-1}\right)^{*}(\mathcal{F})$ also acquires a natural structure of a $\mathbf{T}^{\vee}$-equivariant coherent sheaf, which is does not depend on the choice of $\bar{w}$ up to an isomorphism. Hence $\left(\bar{w}^{-1}\right)^{*}(\mathcal{F})$ defines an element in $K_{0}^{\mathbf{T}^{\vee}}(Y)$. It is easy to see that this element depends only on the image of $\mathcal{F}$ in $\left(\bar{w}^{-1}\right)^{*}(\mathcal{F})$. Hence we get an action of $W$ on $\left(\bar{w}^{-1}\right)^{*}(\mathcal{F})$.

Applying this now to $Y=\mathcal{B}$ we see that the space $K_{0}^{\mathbf{T}^{\vee} \times \mathbb{C}^{*}}(\mathcal{B})$ acquires an action of the algebra $\mathcal{H} \otimes \mathbb{C}[\Gamma \rtimes W]$, where $\mathbb{C}[\Gamma \rtimes W]$ denotes the group algebra of $\Gamma \rtimes W$.

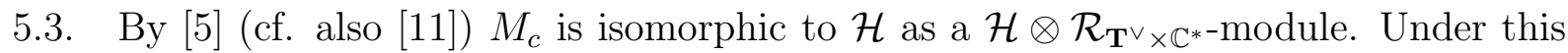
isomorphism the element $1 \in \mathcal{H}$ goes to $A^{+} \in M_{c}$, where $A^{+}$is the unique alcove, which is contained in $\mathcal{C}^{+}$and contains 0 in its closure.

Let

$$
2 \rho^{\vee}=\sum_{\alpha^{\vee} \in \Delta_{+}^{\vee}} \alpha^{\vee}
$$

Consider now the set of fixed points of $\mathbf{T}^{\vee}$ on $\mathcal{B}$. These fixed points are in natural oneto-one correspondence with the elements of $W$. For any $w \in W$ we will denote by $y_{w} \in \mathcal{B}$ the corresponding fixed point. Let $\kappa_{w}$ denote the skyscraper sheaf at $y_{w}$. We let $\mathbf{T}^{\vee}$ act on the fiber of $\kappa_{w}$ by the character $w\left(2 \rho^{\vee}\right)$. We will denote the image of $\kappa_{w}$ in $\mathcal{K}$ by the same symbol. Set $\kappa=\kappa_{e}$.

Following [7] we define a homomorphism $\zeta: M_{c} \rightarrow \mathcal{K}$ of $\mathcal{H} \otimes \mathcal{R}_{\mathbf{T}^{\vee} \times \mathbb{C}^{*} \text {-modules by }}$ sending $A^{+}$to $\kappa$. By [7](Theorem 6.2) $\zeta$ is well-defined and it becomes an isomorphism after tensoring with the ring of fractions of $\mathcal{R}_{\mathbf{T}^{\vee} \times \mathbb{C}^{*}}$. More precisely the following result holds:

Theorem 5.4. (cf. Theorem 6.2 in [7]) There exists $N \in \mathbb{N}$ such that $\mathbf{d}_{v}^{N} \mathcal{K} \subset \zeta\left(M_{c}\right)$ (in fact, in this it is known that one can take $N=1$ but we are not going to use this).

Hence we see that the image of $\zeta^{-1}$ (which a priori) maps $\mathcal{K}$ into some localization of $M_{c}$ ) lies in $M_{\geq}$.

The main result of this section is the following 
Theorem 5.5. 1. The morphism $\zeta$ is " $W$-equivariant", i.e.

$$
\theta_{\alpha}=\zeta^{-1} \circ\left(s_{\alpha}\right)^{*} \circ \zeta
$$

2.

$$
\sum_{w \in W} w^{*}\left(\zeta\left(M_{c}\right)\right)=\mathcal{K}
$$

Corollary 5.6. The map $\zeta$ extends to an isomorphism between $M_{d}$ and $\mathcal{K}$.

This follows immediately from Theorem 5.5 and Proposition 4.5.

Corollary 5.7. $\mathcal{K}_{q}$ is naturally isomorphic to $\mathcal{S}(X)^{I \times \mathbf{T}(\mathcal{O})}$ as a $\mathcal{H} \otimes \mathbb{C}[\widetilde{W}]$-module.

Note that we do not need Corollary 4.6 in order to prove Corollary 5.7.

5.8. Proof of Theorem 5.5. As in [11] we can regard both $M_{c}$ and $\mathcal{K}$ as sections of some vector bundles $\mathbf{V} \rightarrow \mathbf{T}^{\vee} \times \mathbb{C}^{*}$ and $\mathbf{V}^{\prime} \rightarrow \mathbf{T}^{\vee} \times \mathbb{C}^{*}$. The map $\zeta$ induces a meromorphic isomorphism between $\mathbf{V}$ and $\mathbf{V}^{\prime}$. Now both the left hand side and the right hand side of (5.2) are meromorphic maps from $\mathbf{V}$ to itself, which induce the same map $s_{\alpha}$ on the base. On the other hand, it is well known that the fiber of $\mathbf{V}$ at the generic point of $\mathbf{T}^{\vee} \times \mathbb{C}^{*}$ is an irreducible representation of $\mathcal{H}$. Hence the left hand side and the right hand side of (5.2) differ by multiplication by certain meromorphic function $f$ on $\mathbf{T}^{\vee} \times \mathbb{C}^{*}$.

On the other hand we know that $\theta_{\alpha}^{2}=\left(\zeta^{-1} \circ s_{\alpha}^{*} \circ \zeta\right)^{2}=\mathrm{id}$. This implies that

$$
f(\mathbf{t}, \lambda) f\left(s_{\alpha}(\mathbf{t}), \lambda\right)=1
$$

We claim now that $f(\mathbf{t}, 1)=1$ for every $\mathbf{t} \in \mathbf{T}^{\vee}$ (it is easy to see that the divisor $v=1$ is not a singular divisor for $f$, hence the restriction of $f$ to $v=1$ makes sense). To prove this we must show that $\zeta$ commutes with the action of $W$ when we specialize to $v=1$. We will denote the corresponding specializations by $\zeta^{1}, M_{c}^{1}, \mathcal{K}^{1}$ etc. (Note that for $v=1$ the operators $\theta_{\alpha}^{1}$ preserve $M_{c}^{1}$ and therefore define an action of $W$ there). By (4.3) one has $T_{w}^{1} A^{+}=A^{+} w$. It is easy to see that one also has $T_{\alpha}^{1} \kappa=s_{\alpha}(\kappa)$. Indeed, fix $\alpha \in \Pi$. Let $\pi_{\alpha}: \mathcal{B} \rightarrow \mathcal{B}_{\alpha}$ denote the projection from $\mathcal{B}$ to the corresponding partial flag variety. Let also $\Omega_{\alpha}$ denote the corresponding sheaf of relative differential forms. Then

$$
T_{\alpha}^{1}(\mathcal{F})=\pi_{\alpha}^{*} \circ\left(\pi_{\alpha}\right)_{*}(\mathcal{F})-\pi_{\alpha}^{*} \circ\left(\pi_{\alpha}\right)_{*}\left(\mathcal{F} \otimes \Omega_{\alpha}\right)-\mathcal{F}
$$

Let $\mathcal{P}=\pi_{\alpha}^{-1}\left(\pi_{\alpha}\left(y_{e}\right)\right)=\pi_{\alpha}^{-1}\left(\pi_{\alpha}\left(y_{s_{\alpha}}\right)\right.$. Then $\mathcal{P}$ is $\mathbf{T}^{\vee}$-stable and therefore the structure sheaf $\mathcal{O}_{\mathcal{P}}$ acquires the natural $\mathbf{T}^{\vee}$-equivariant structure. Also $\mathcal{P}$ is $\mathbf{T}^{\vee}$-equivariantly isomorphic to the projective line $\mathbb{P}^{1}$, where we let $\mathbf{T}^{\vee}$ act on $\mathbb{P}^{1}$ by means of the character $\alpha^{\vee}: \mathbf{T}^{\vee} \rightarrow \mathbb{C}^{*}$, composed with the standard $\mathbb{C}^{*}$-action on $\mathbb{P}^{1}$. For any $\gamma \in \widetilde{\Gamma}$ and $n \in \mathbb{Z}$, such that $n+\langle\alpha, \gamma\rangle$ is even, we will denote by $\mathcal{O}(n)_{\mathcal{P}}^{\gamma}$ the image in $\mathcal{K}^{1}$ of the bundle 
$\mathcal{O}(n)_{\mathcal{P}} \otimes \mathbb{C}_{\gamma}$ (where $\mathbb{C}_{\gamma}$ is the one-dimensional representation of a covering of $\mathbf{T}^{\vee}$, on which the Lie algebra $\mathfrak{t}^{\vee}$ acts by means of $d \gamma$ ). Thus

$$
T_{\alpha}^{1}(\kappa)=\mathcal{O}_{\mathcal{P}}^{2 \rho^{\vee}}+\mathcal{O}_{\mathcal{P}}^{2 \rho^{\vee}-2 \alpha^{\vee}}-\kappa
$$

. But it is easy to see that (note also that $2 \rho^{\vee}-2 \alpha^{\vee}=s_{\alpha}\left(\rho^{\vee}\right)$ )

$$
\mathcal{O}_{\mathcal{P}}^{2 \rho^{\vee}}-\kappa=\mathcal{O}_{\mathcal{P}}^{s_{\alpha}\left(2 \rho^{\vee}\right)}-\kappa\left(y_{s_{\alpha}}\right)=\mathcal{O}(-1)_{\mathcal{P}}^{\rho^{\vee}}
$$

Hence $T_{\alpha}^{1}(\kappa)=\kappa\left(y_{s_{\alpha}}\right)=s_{\alpha}(\kappa)$. Thus $T_{w}^{1} \kappa=w(\kappa)$ for every $w \in W$. This implies that $f(\mathbf{t}, 1)=1$.

On the other hand, it follows from the above, that $f$ must be of the form $f=\chi \mathbf{d}_{v}^{N}$, where $\chi$ is a regular invertible function on $\mathbf{T}^{\vee} \times \mathbb{C}^{*}$, for some integer $N$. Thus

$$
1=f s_{\alpha}^{*}(f)=\chi s_{\alpha}^{*}(\chi) \mathbf{d}_{v}^{N} s_{\alpha}^{*}\left(\mathbf{d}_{v}^{N}\right)
$$

which implies that $\mathbf{d}_{v}^{N} s_{\alpha}^{*}\left(\mathbf{d}_{v}^{N}\right)$ is regular and invertible. It is easy to see that this may happen only for $N=0$.

Thus $f=\chi$ is regular and invertible. But then $f$ is just a scalar multiple of a character of $\mathbf{T}^{\vee} \times \mathbb{C}^{*}$ and therefore (5.4) and the fact that $f(\mathbf{t}, 1)=1$ for every $\mathbf{t} \in \mathbf{T}^{\vee}$ implies that $f$ must be identically equal to 1 . Hence (5.2) holds.

We claim now that Theorem 5.5(2) follows immediately from Theorem 5.4. Indeed, arguing in the same way as in Lemma 3.15 we can show that the ideal in $\mathcal{R}_{\mathbf{T}^{\vee} \times \mathbb{C}^{*}}$ generated by the functions $\left\{w\left(\mathbf{d}_{v}^{N}\right)\right\}_{w \in W}$ contains 1, which implies Theorem 5.5(2) in view of Theorem 5.4.

\section{Description of the DUAL SPACE}

6.1. Let $\mathcal{S}^{\prime}(X)$ denote the dual space to $\mathcal{S}(X)$. Let also $\mathcal{D}(X)$ denote the space of distributions on $X$ (i.e. dual space to $\mathcal{S}_{c}(X)$ ). Clearly $W$ acts on $\mathcal{S}^{\prime}(X)$ and we will denote the corresponding operators also by $\Phi_{w}$. Also one has a surjective map $r: \mathcal{S}^{\prime}(X) \rightarrow \mathcal{D}(X)$. The main result of this section is the following theorem, which will be used in the next subsection where we discuss a global analogue of $\mathcal{S}(X)$.

First of all let us introduce some notations. For every simple root $\alpha$ of $G$ let

$$
\mathcal{S}_{\alpha}^{0}(X)=\left\{f \in \mathcal{S}_{c}(X) \mid \text { such that } \Phi_{\alpha}(f) \in \mathcal{S}_{c}(X)\right\}
$$

Theorem 6.2. The space $\mathcal{S}^{\prime}(X)$ is isomorphic to the space of all collections $\left\{\lambda_{w}\right\}_{w \in W}$ where $\lambda_{w} \in \mathcal{D}(X)$ satisfying the following condition

$$
\lambda_{s_{\alpha} w}(f)=\lambda_{w}\left(\Phi_{s_{\alpha}}(f)\right) \quad \text { for any } w \in W, \alpha \in \Pi, f \in \mathcal{S}_{\alpha}^{0}(X)
$$

Moreover, this isomorphism can be described by sending $\lambda \in \mathcal{S}^{\prime}(X)$ to $\left\{\lambda_{w}\right\}$, where $\lambda_{w}=r\left(\Phi_{w}(\lambda)\right)$. 
Proof. Remark 6.3. Note the similarity between Theorem 6.2 and the main result of [3].

Choose $\alpha \in \Pi$. Let us denote by $W^{\alpha}$ the subset of $W$, consisting of all minimal representatives of $W /\left\{1, s_{\alpha}\right\}$.

Consider the complex $K^{2} \rightarrow K^{1} \rightarrow K^{0}$ where

- $K^{0}=\mathcal{S}(X)$

- $K^{1}=\oplus_{w \in W} \mathcal{S}_{c}(X)$

- $K^{2}=\oplus_{\alpha \in \Pi, w \in W^{\alpha}} \mathcal{S}_{\alpha}^{0}(X)$

where the differential $d: K^{1} \rightarrow K^{0}$ is given by

$$
d\left(\left\{f_{w}\right\}\right)=\sum_{w \in W} \Phi_{w}\left(f_{w}\right)
$$

and the differential $d: K^{2} \rightarrow K^{1}$ is given by the following formula. Suppose that we are given an element $k \in K^{2}$, whose $(w, \alpha)$-component is equal to $f$ for some $\alpha \in \Pi, w \in W^{\alpha}$ and $f \in \mathcal{S}_{\alpha}^{0}(X)$. Then $d(k)$ is defined by

$$
d(k)_{w}=f, d(k)_{w s_{\alpha}}=-\Phi_{\alpha}(f), d(k)_{w^{\prime}}=0 \text { otherwise }
$$

It is easy to see now that the assertion of Theorem 6.2 is equivalent to the fact that the above complex is exact in the middle term.

6.4. The complex $C^{*}(M)$. Let $\mathfrak{t}_{\mathbb{R}}=\Gamma \otimes \mathbb{R}$ be a real Cartan algebra of $\mathbf{G}$. Define a convex polytope $B$ in $\mathfrak{t}_{\mathbb{R}}$ in the following way. Choose a regular point $a \in \mathfrak{t}$ and define $B$ to be the convex hull of the set $w(a), w \in W$.

For any $I \subset \Pi$ let $W^{I}$ denote the set of all minimal representatives of $W / W_{I}$, where $W_{I}$ is the subgroup of $W$ generated by $s_{i}$ for $i \in I$. Then $B$ admits a natural cell decomposition such that the cells of dimension $i$ are parametrized by all pairs $\left(I \subset \Pi, w \in W^{I}\right)$ such that $|I|=i$. Let $C^{*}(B)$ denote the "augmented" cohomology complex associated with this cell decomposition. This means that $C^{i}(B)$ is the space with a basis parametrized by cell of dimension $i$ of $B$ and $C^{0}(B)=\mathbb{C}$ with the natural differential $d: C^{i} \rightarrow C^{i+1}$. The complex $C^{*}(B)$ is obviously acyclic, since $B$ is contractible.

Let $M$ be any $W$-module. Then we can define a complex $\left(C^{*}(M), d_{M}\right)$ in the following manner. First of all we set $C^{i}(M)=M \otimes C^{i}(B)$. Let now $m \otimes c \in C^{i}(M)$. Suppose that

$$
d(c)=\sum_{w \in W, \alpha \in \Pi \backslash I} c_{w, I \cup\{\alpha\}}
$$

(here $c_{w, I \cup\{\alpha\}}$ denotes the corresponding component of $d(c)$ ). Then we define

$$
d_{M}(m \otimes c)=\sum_{w \in W, \alpha \in \Pi \backslash I} s_{\alpha}(m) \otimes c_{w, I \cup\{\alpha\}}
$$

The complex $\left(C^{*}(M), d_{M}\right)$ is still acyclic because its differential is conjugate to id $\otimes d$. 
6.5. End of the proof. Recall that we have to prove that the complex $K^{2} \rightarrow K^{1} \rightarrow K^{0}$ is acyclic in the middle term. But it follows from Theorem 3.8 and Theorem 3.11 that this is equivalent to showing that $\left(K^{0}\right)^{\vee} \rightarrow\left(K^{1}\right)^{\vee} \rightarrow\left(K^{2}\right)^{\vee}$ is acyclic in the middle term (we use here the fact that $K^{1}$ and $K^{2}$ are $\mathcal{A}$-reflexive; note that we don't need to know that $K^{0}$ is $\mathcal{A}$-reflexive).

However, it follows again from Theorem 3.8 and Theorem 3.11 that the latter complex embeds naturally into the complex $C^{*}(\mathcal{S}(X))$, which is acyclic by Section 6.4. Let now $x \in\left(K^{1}\right)^{\vee}$ be such that $d(x)=0$. Then there exists some $y \in \mathcal{S}(X)$ such that $d_{\mathcal{S}(X)}(y)=$ $x$. However $d_{\mathcal{S}(X)}(y)=\oplus_{w \in W} \Phi_{w}(y)$. On the other hand it follows once more from Theorem 3.8 that $\left(K^{1}\right)^{\vee}=\oplus_{w \in W} \mathcal{S}_{c}(X)$. Hence we see that $\Phi_{w}(y) \in \mathcal{S}_{c}(X)$ for every $w \in W$. This means that $y \in \mathcal{S}^{0}(X)=\left(K^{0}\right)^{\vee}$ which finishes the proof.

\section{The Global SPACE}

7.1. Remarks on arcimedean places. We now want to describe some global analogue of the space $\mathcal{S}(X)$. In order to do that we will need a definition of $\mathcal{S}(X)$ in the case where the base local field $k$ is arcimedian. So, let $\mathbf{G}$ be a semisimple connected simply connected algebraic group over $k=\mathbb{R}, \mathbb{C}$. We will assume in fact that $k=\mathbb{R}$ (which can be done without loss of generality) and that $\mathbf{G}$ is quasi-split over $k$. Clearly the space $L^{2}(X)$ still makes sense and one still has an action of $W$ on this space defined exactly as before. Let $\mathcal{D}$ denote the algebra of all global sections of the sheaf of regular differential operators on $\mathbf{X}$. Thus we define

$$
\mathcal{S}(X)=\left\{f \in L^{2}(X) \mid \text { such that } d(f) \in L^{2}(X) \text { for every } d \in \mathcal{D}\right\}
$$

It is easy to see that $\mathcal{S}(X)$ is invariant under the operators $\Phi_{w}$.

In the sequel we will need the following estimate on the growth rate of elements of $\mathcal{S}$. Choose a maximal compact subgroup $K_{\mathbb{R}}$ of $G=\mathrm{G}(\mathbb{R})$. One can choose a Borel subgroup $\mathbf{B}$ of $\mathbf{G}$ in such a way that $G=K_{\mathbb{R}} \cdot B$, where $B=\mathbf{B}(\mathbb{R})$. Hence the natural map $\phi: \mathbf{T}(\mathbb{R})=T \rightarrow X$ becomes surjective after we quotient out $X$ by $K_{\mathbb{R}}$. Let also $\xi_{\mathbb{R}}: T \rightarrow \mathfrak{t}_{\mathbb{R}}$ denote the homomorphism of abelian groups, defined by the condition

$$
\left\langle\lambda^{\vee}, \xi_{\mathbb{R}}(t)\right\rangle=\ln \left|\lambda^{\vee}(t)\right|
$$

for every weight $\lambda^{\vee} \in \Gamma^{\vee}$.

Lemma 7.2. Let $f \in \mathcal{S}(X)$. Then for every $n \in \mathbb{N}$ there exists a real number $c=c(n)$ such that

$$
|f(\phi(t))|<c\left|\left\langle\rho, \xi_{\mathbb{R}}(t)\right\rangle\right|^{-n}
$$

The proof is left to the reader. 
7.3. More notations. Let now $F$ be a global field and let $\mathbf{G}$ be again a semisimple simply connected algebraic group defined over $F$. Let also $\mathbb{A}_{F}$ denote the ring of adeles of $F$. Let $v$ be a place of $F$ and let $F_{v}$ denote the corresponding local field.

In this case the variety $X$ is defined over $F$. We will suppose that the symplectic forms $\omega_{\alpha}$ (cf. Section 2.1) are also $F$-rational.

Also we will fix a non-trivial character $\psi: \mathbb{A}_{F} / F \rightarrow \mathbb{C}^{*}$. This character defines a nontrivial character $\psi_{v}$ of $F_{v}$ for every place $v$. All the local Fourier transforms that we are going to consider will be with respect to the characters $\psi_{v}$.

7.4. The global space. Let $v$ be a place of $F$. Then we may consider the space $\mathcal{S}_{v}=$ $\mathcal{S}\left(\mathbf{X}\left(F_{v}\right)\right)$. By Theorem 3.13 the space $\mathcal{S}_{v}$ has distinguished $K_{v}=\mathbf{G}\left(\mathcal{O}_{v}\right) \times \mathbf{T}\left(\mathcal{O}_{v}\right)$ invariant vector $c_{0}^{v}$ (we do not define $e_{0}^{v}$ when $v$ is archimedean).

Definition 7.5. The space $\mathcal{S}$ is the restricted tensor product of the spaces $\mathcal{S}_{v}$ (over all places $v$ of $F$ ) with respect to the vectors $c_{0}^{v}$. We will also endow $\mathcal{S}$ with a topology, which comes from the discrete topology on $\mathcal{S}\left(\mathbf{X}\left(F_{v}\right)\right.$ for non-arcimedean $v$ and from Frechet topology on $\mathcal{S}\left(\mathbf{X}\left(F_{v}\right)\right.$ for archimedean $v$.

Since the vector $c_{0}^{v} \in \mathcal{S}_{v}$ is $W$-invariant, it follows that the $W$-action extends (diagonally) to the space $\mathcal{S}$. As before for any $w \in W$ we let $\Phi_{w}$ denote the corresponding operator. On the other hand, it is clear that $\mathcal{S}$ also admits a natural smooth action of the group $\mathbf{G}\left(\mathbb{A}_{F}\right) \times \mathbf{T}\left(\mathbb{A}_{F}\right)$ compatible in the obvious sense with the action of $W$.

7.6. The functional $\varepsilon_{0}$. We claim now that there exists a natural morphism from $\mathcal{S}$ to the space of smooth functions on $\mathbf{X}\left(\mathbb{A}_{F}\right)$. Indeed if $f=\otimes f_{v} \in \mathcal{S}$ and $x=\left\{x_{v}\right\} \in \mathbf{X}\left(\mathbb{A}_{F}\right)$ then we define

$$
f(x)=\prod f_{v}\left(x_{v}\right)
$$

It is easy to see that the product in the left hand side of (7.4) is actually finite (this follows from the fact that $x_{v} \in X_{0, v}$ for almost any $v$ ).

We define now

$$
\varepsilon_{0}(f)=\sum_{x \in \mathbf{X}(F) \subset \mathbf{X}\left(\mathbb{A}_{F}\right)} f(x)
$$

It is clear that the functional $\varepsilon_{0}$ on $\mathcal{S}$ is $\mathbf{G}(F) \times \mathbf{T}(F)$ - invariant, but not $W$-invariant. Our next goal is to produce some natural $W$-invariant modification of $\varepsilon$.

7.7. The spaces $\mathcal{S}_{c, v}$. Fix a place $v$ of $F$. Let $\mathcal{S}_{c, v}$ denote the subspace of $\mathcal{S}$ spanned by all functions of the form $\otimes f_{v^{\prime}}$ where $f_{v} \in \mathcal{S}_{c}\left(\mathbf{X}\left(F_{v}\right)\right)$. Also, for any $\alpha \in \Pi$ let

$$
\mathcal{S}_{\alpha, v}^{0}=\left\{f \in \mathcal{S}_{c, v} \mid \Phi_{\alpha}(f) \in \mathcal{S}_{c, v}\right\}
$$

Set also $\mathcal{S}_{\alpha}^{0}=\sum_{v} \mathcal{S}_{\alpha, v}^{0}$. 
Proposition 7.8. For any simple root $\alpha$ and any $f \in \mathcal{S}_{\alpha}^{0}$ one has

$$
\varepsilon_{0}(f)=\varepsilon_{0}\left(\Phi_{\alpha}(f)\right)
$$

Proof. Suppose first that $G=S L(2)$. In this case $\mathcal{S}$ is the space of smooth compactly supported functions on $\mathbb{A}_{F}^{2}$ and the space $\mathcal{S}_{\alpha}^{0}$ consists of all functions $f$ such that

$$
f(0)=\Phi(f)(0)=0
$$

(here we have set $\Phi=\Phi_{\alpha}$ ). Also one has

$$
\varepsilon_{0}(f)=\sum_{x \in F^{2}, x \neq 0} f(x)
$$

It follows now from the Poisson summation formula that for any $f \in \mathcal{S}$ one has

$$
\sum_{x \in F^{2}} f(x)=\sum_{x \in F^{2}} \Phi(f)(x)
$$

Now it is easy to see that (7.8), (7.9) and (7.10) imply together that $\varepsilon_{0}(f)=\varepsilon_{0}(\Phi(f))$ for any $f \in \mathcal{S}_{\alpha}^{0}$ which proves Proposition 7.8 for $\mathbf{G}=S L(2)$.

In the general case Proposition 7.8 follows from the same arguments as above and the following result.

Proposition 7.9. Let $p: \mathbf{E} \rightarrow \mathbf{Y}$ be a symplectic vector bundle, defined over a global field $F$. Let $f$ be any function on $\mathbf{E}\left(\mathbb{A}_{F}\right)$ which satisfies the following condition:

for every $y \in \mathbf{Y}\left(\mathbb{A}_{F}\right)$ the restriction of $f$ to $p^{-1}(y)$ lies in the space of Schwartz-Bruhat functions of $p^{-1}(y)$.

The the Fourier transform $\Phi(f)$ is well-defined, and, moreover, one has

$$
\sum_{x \in E(F)} f(x)=\sum_{x \in E(F)} \Phi(f)(x)
$$

Theorem 7.10. There exists unique $W$-invariant functional $\varepsilon$ on $\mathcal{S}$ such that for any place $v$ of $F$ the restriction of $\varepsilon$ to $\mathcal{S}_{c, v}$ coincides with the restriction of $\varepsilon_{0}$ to $\mathcal{S}_{c, v}$. The functional $\varepsilon$ is $\mathbf{G}(F) \times \mathbf{T}(F)$-invariant.

Proof. The uniqueness of $\varepsilon$ is obvious. Namely, let $f \in \mathcal{S}$. Choose a place $v$ of $F$. Then there exist functions $f_{w} \in \mathcal{S}_{c, v}$ such that

$$
f=\sum_{w \in W} \Phi_{w}\left(f_{w}\right)
$$

We set now

$$
\varepsilon(f)=\sum_{w \in W} \varepsilon\left(f_{w}\right)
$$


(It is clear that if $\varepsilon$ exists then it must satisfy (7.13)).

In order to show that $\varepsilon$ is well-defined, we must show that the right hand of $(7.13)$ does not depend on the choice of the functions $f_{w}$ and on the choice of $v$. However, the first statement follows immediately from Theorem 6.2 and the second statement follows from the following lemma, whose proof is exactly the same as the proof of Theorem 6.2

Proposition 7.11. Let $v_{1}, v_{2}$ be two places of $F$. Let $V=\mathcal{S}\left(\mathbf{X}\left(F_{v_{1}}\right)\right) \otimes \mathcal{S}\left(\mathbf{X}\left(F_{v_{2}}\right)\right)$. The group $W$ acts on $V$ diagonally. Set also

$$
V_{c}=\mathcal{S}_{c}\left(\mathbf{X}\left(F_{v_{1}}\right)\right) \otimes \mathcal{S}\left(\mathbf{X}\left(F_{v_{2}}\right)\right)+\mathcal{S}_{c}\left(\mathbf{X}\left(F_{v_{1}}\right)\right) \otimes \mathcal{S}\left(\mathbf{X}\left(F_{v_{2}}\right)\right)
$$

Set $V_{\alpha}^{0}=V_{c} \cap \Phi_{\alpha}\left(V_{c}\right)$. Let also $V_{c}^{\prime}$ denote the dual space to $V_{c}$.

Suppose now that we are given a collection $\left\{\lambda_{w}\right\}_{w \in W}$, where $\lambda_{w} \in V_{c}^{\prime}$, such that $\lambda_{w}(f)=$ $\lambda_{s_{\alpha} w}\left(\Phi_{\alpha}\left(f_{s_{\alpha} w}\right)\right)$ for any $f \in V_{\alpha}^{0}$. Then $\left\{\lambda_{w}\right\}$ gives rise to a well defined functional on $V$.

7.12. Let $Y_{\mathbf{T}}=\left(\mathbf{G}\left(\mathbb{A}_{F}\right) \times \mathbf{T}\left(\mathbb{A}_{F}\right)\right) /(\mathbf{G}(F) \times \mathbf{T}(F))$ and let $C^{\infty}\left(Y_{\mathbf{T}}\right)$ denote the space of smooth functions on $Y_{\mathbf{T}}$. Thus we may define may $\mathbf{G}\left(\mathbb{A}_{F}\right) \times \mathbf{T}\left(\mathbb{A}_{F}\right) \rtimes W$ - equivariant map $\eta: \mathcal{S} \rightarrow C^{\infty}\left(Y_{\mathbf{T}}\right)$ by

$$
\eta(f)(g, t)=\varepsilon((g \times t)(f))
$$

where $(g \times t)(f)(x)=f\left(g^{-1} x t^{-1}\right)$. Let also $\eta_{0}$ be the corresponding map, defined as in (7.15) but with $\varepsilon$ replaced by $\varepsilon_{0}$.

7.13. Let now $\chi$ be a character of $\mathbf{T}\left(\mathbb{A}_{F}\right) / \mathbf{T}(F)$. We wish to define a map $E_{\chi}$ from $\mathcal{S}$ to the space of functions on $\mathbf{G}\left(\mathbb{A}_{F}\right) / \mathbf{G}(F)$ by putting

$$
E_{\chi}(f)(g)=\int_{\mathbf{T}\left(\mathbb{A}_{F}\right) / \mathbf{T}(F)} \eta(f)(g, t) \chi(t) d t
$$

However, the integral in the right hand side of (7.16) does not have to converge and we will have to regularize it. We will do that only for regular characters $\chi$ (cf. Definition 7.14 below). In fact, for any regular character we will define a morphism of $\mathbf{G}\left(\mathbb{A}_{F}\right) \times \mathbf{T}\left(\mathbb{A}_{F}\right)$ modules $E_{\chi}: \mathcal{S} \rightarrow C^{\infty}\left(\mathbf{G}\left(\mathbb{A}_{F}\right) / \mathbf{G}(F)\right.$, where $\mathbf{T}\left(\mathbb{A}_{F}\right)$ acts on $C^{\infty}\left(\mathbf{G}\left(\mathbb{A}_{F}\right) / \mathbf{G}(F)\right.$ by means of $\chi$, which "morally" will be the regularization of the above integral.

Let $\chi$ be a character of $\mathbf{T}\left(\mathbb{A}_{F}\right) / \mathbf{T}(F)$ and let $w \in W$. Then we set $w_{\bullet} \chi=\|\cdot\|^{-1} w(\chi\|\cdot\|)$.

Definition 7.14. We will say that $\chi$ is regular if for any corroot $\alpha^{\vee}: \mathbb{G}_{m} \rightarrow \mathbf{T}$ and any $w \in W$, the induced character $w_{\bullet} \chi \circ \alpha^{\vee}$ of $\mathbb{A}_{F}^{*} / F^{*}$ is non-trivial.

Note that the set of regular characters is invariant under the action of $W$. 
7.15. Regularization of the integral for regular characters. Let $\mathbb{A}_{F}^{*} / F^{*}$ be the idele class group of $F$. Then we have the norm map $\|\cdot\|: \mathbb{A}_{F}^{*} / F^{*} \rightarrow \mathbb{R}^{+}$.

Consider now the group $\mathbf{T}\left(\mathbb{A}_{F}\right) / \mathbf{T}(F)$. Recall that $\mathfrak{t}_{\mathbb{R}}$ denote the real Cartan algebra of $\mathbf{G}$ (i.e. $\mathfrak{t}_{\mathbb{R}}=\Gamma \otimes \mathbb{R}$. Then there exists unique homorphism of abelian groups $\xi: \mathbf{T}\left(\mathbb{A}_{F}\right) / \mathbf{T}(F) \rightarrow \mathfrak{t}_{\mathbb{R}}$ satisfying

$$
\left\langle\lambda^{\vee}, \xi(t)\right\rangle=\ln \left\|\lambda^{\vee}(t)\right\|
$$

for any $t \in \mathbf{T}\left(\mathbb{A}_{F}\right) / \mathbf{T}(F)$ and $\lambda^{\vee} \in \Gamma^{\vee}$ (note that $\lambda^{\vee}$ defines a map $\mathbf{T}\left(\mathbb{A}_{F}\right) / \mathbf{T}(F) \rightarrow$ $\mathbb{A}_{F}^{*} / F^{*}$ which, abusing the notations, we denote by the same letter).

Let

$$
\mathfrak{t}_{\mathbb{R}}=\bigcup_{w \in W} \mathfrak{t}_{\mathbb{R}}^{w}
$$

be the decomposition of $\mathfrak{t}_{\mathbb{R}}$ into Weyl chambers.

Proposition 7.16. For any $f \in \mathcal{S}$ the integral

$$
\int_{\xi^{-1}\left(\mathfrak{t}_{\mathbb{R}}^{e}\right)} \eta_{0}(f)(g, t) \chi(t) d t
$$

is absolutely convergent. Here $e \in W$ denotes the unit element.

Proof. Proposition 7.16 follows immediately from the following lemma.

Lemma 7.17. Let $f \in \mathcal{S}$. Then for any $g \in \mathbf{G}\left(\mathbb{A}_{F}\right) / \mathbf{G}(F)$ and $n \in \mathbb{N}$ there exists a real number $c=c(g, n)$ such that

$$
\left|\eta_{0}(f)(g, t)\right|<c|\langle\rho, \xi(t)\rangle|^{-n}
$$

The lemma follows easily from Lemma 7.2 and Lemma 3.3 .

Suppose now that $\chi$ is a regular character of $\mathbf{T}\left(\mathbb{A}_{F}\right) / \mathbf{T}(F)$. Then we can define the $\operatorname{map} E_{\chi}: \mathcal{S} \rightarrow C^{\infty}\left(\mathbf{G}\left(\mathbb{A}_{F}\right) / \mathbf{G}(F)\right)$ by

$$
E_{\chi}(f)(g)=\sum_{w \in W_{\xi^{-1}\left(\mathfrak{t}_{\mathbb{R}}^{e}\right)}} \eta_{0}\left(\Phi_{w}\right)(f)(g, t) w_{\bullet}(\chi(t)) d t
$$

Proposition 7.18. 1. $E_{\chi}$ is a $\mathbf{G}\left(\mathbb{A}_{F}\right) \times W$-equivariant map (where $W$ acts trivially on $C^{\infty}\left(\mathbf{G}\left(\mathbb{A}_{F}\right) / \mathbf{G}(F)\right)$.

2. Let $\mathcal{S}_{\chi}$ denote the space of $\left(\mathbf{T}\left(\mathbb{A}_{F}\right), \chi\right)$ - coinvariants on $\mathcal{S}$, i.e.

$$
\mathcal{S}_{\chi}=\mathcal{S} / \overline{\operatorname{span}}\left(\chi(t) g-t(g) \mid g \in \mathcal{S}, t \in \mathbf{T}\left(\mathbb{A}_{F}\right)\right)
$$

(here the bar denotes the closure of the corresponding subspace). Then $E_{\chi}$ descends to a well-defined map Eis E $_{\chi} \rightarrow C^{\infty}\left(\mathbf{G}\left(\mathbb{A}_{F}\right) / \mathbf{G}(F)\right)$. 
Remark 7.19. We will see some connection between Eis $_{\chi}$ and Eisenstein series in the next subsection.

Proof. The second statement of Proposition 7.18 is clear from the definitions. Let us prove the first one.

The $\mathbf{G}\left(\mathbb{A}_{F}\right)$-equivariance of $E_{\chi}$ is obvious. Let us prove that $E_{\chi}$ is $W$-equivariant, i.e. that $E_{\chi}(f)=E_{\chi}\left(\Phi_{w}(f)\right)$ for every $f \in \mathcal{S}, w \in W$. Clearly, it is enough to show this when $w=s_{\alpha}$ is a simple reflection. Hence Proposition 7.18 follows from the following lemma.

Lemma 7.20. For every $f \in \mathcal{S}$ and any regular character $\chi$ one has

$$
\int_{\xi^{-1}\left(\mathfrak{t}_{\mathbb{R}}^{e}\right)} \eta_{0}(f)(g, t)(\chi(t)) d t=\int_{\xi^{-1}\left(\mathfrak{t}_{\mathbb{R}}^{e}\right)} \eta_{0}\left(\Phi_{\alpha}(f)\right)(g, t) s_{\alpha}(\chi(t)) d t
$$

Proof. (Of Lemma 7.20). Recall that for every simple root $\alpha$ we have defined the $\mathbf{G}\left(\mathbb{A}_{F}\right) \times$ $\mathbf{T}\left(\mathbb{A}_{F}\right)$-submodule $\mathcal{S}_{\alpha}^{0}$ of $\mathcal{S}$ which is invariant under $\Phi_{\alpha}$. Moreover, by Proposition 7.8, one has

$$
\eta_{0}(f)=\eta_{0}\left(\Phi_{\alpha}(f)\right)
$$

for every $f \in \mathcal{S}_{\alpha}^{0}$. Therefore, Lemma 7.20 holds for every $f \in \mathcal{S}_{\alpha}^{0}$. Hence Lemma 7.20 follows from the following easy lemma.

Lemma 7.21. The natural map $\left(\mathcal{S}_{\alpha}^{0}\right)_{\chi} \rightarrow \mathcal{S}_{\chi}$ is an isomorphism.

7.22. Connection with usual Eisenstein series. Let

$$
\pi_{\chi}=\operatorname{Ind}_{\mathbf{B}\left(\mathbb{A}_{F}\right)}^{\mathbf{G}\left(\mathbb{A}_{F}\right)} \chi
$$

where we regard $\chi$ as a character of $\mathbf{B}\left(\mathbb{A}_{F}\right)$ by means of the identification $\mathbf{B}\left(\mathbb{A}_{F}\right) / \mathbf{U}\left(\mathbb{A}_{F}\right)=$ $\mathbf{T}\left(\mathbb{A}_{F}\right)$. Then by [8] one has the Eisenstein series map $\operatorname{Eis}_{\chi}^{\prime}: \pi_{\chi} \rightarrow C^{\infty}\left(\mathbf{G}\left(\mathbb{A}_{F}\right) / \mathbf{G}(F)\right)$, which is meromorphic in $\chi$ (in particular, it is well-defined for generic $\chi$ ).

Choose now a finite set of places $P$ of $F$ such that $P$ contains all places, where $\chi$ is ramified and all arcimedean places of $F$. Then we can define a map $i_{P, \chi}: \pi_{\chi} \rightarrow \mathcal{S}_{\chi}$ in the following way.

Let $K_{P}=\prod_{v \notin P} \mathbf{G}\left(\mathcal{O}_{v}\right)$. The representation $\pi_{\chi}$ is generated by its $K_{P}$-invariant vectors. Hence it is enough to construct a map $\pi_{\chi}^{K_{P}} \rightarrow \mathcal{S}_{\chi}^{K_{P}}$ which commutes with the action of the corresponding Hecke algebra.

By the definition of an induced representation, $\pi$ is a quotient of the space $C_{c}^{\infty}\left(\mathbf{X}\left(\mathbb{A}_{F}\right)\right)$ of smooth compactly supported functions on $\mathbf{X}\left(\mathbb{A}_{F}\right)$. Moreover, $\pi_{\chi}^{K_{P}}$ is a quotient of 
$\left(C_{c}^{\infty}\left(\mathbf{X}\left(\mathbb{A}_{F}\right)\right)^{K_{P}}\right.$. On the other hand, we have the natural morphism $l_{P}: \mathcal{S}_{\chi}^{K_{P}} \rightarrow$ $\left(C_{c}^{\infty}\left(\mathbf{X}\left(\mathbb{A}_{F}\right)\right)^{K_{P}}\right.$ sending $\otimes_{v} f_{v}$ to

$$
\bigotimes_{v \in P} f_{v} \otimes \bigotimes_{v \notin P}\left(\prod_{\alpha^{\vee} \in \Delta_{+}^{\vee}}\left(1-q_{v}^{-1} H_{\alpha^{\vee}}\right)\right)\left(f_{v}\right)
$$

where $q_{v}$ is the number of elements in the residue field of $F_{v}$ and $H_{\alpha^{\vee}}$ is as in Section 3.12. Thus composing $l_{P}$ with $\operatorname{Eis}_{\chi}$ we get the map $\operatorname{Eis}_{\chi, P}: \pi_{\chi} \rightarrow C^{\infty}\left(\mathbf{G}\left(\mathbb{A}_{F}\right) / \mathbf{G}(F)\right)$.

Let now

$$
L_{P}(\chi)=\prod_{\alpha^{\vee} \in \Delta_{+}^{\vee}} \prod_{v \notin P}\left(1-q_{v}^{-1+\left\langle\alpha^{\vee}, \rho\right\rangle} \chi_{\alpha^{\vee}}\left(\pi_{v}\right)\right)^{-1}
$$

be the corresponding $L$-function, which is a meromorphic function of $\chi$. Here the notations are the following: $\chi_{\alpha}$ denotes the character of $\mathbb{A}_{F}^{*} / F^{*}$ obtained by composing $\chi$ with the homomorphism $\mathbb{A}_{F}^{*} / F^{*} \rightarrow \mathbf{T}\left(\mathbb{A}_{F}\right) / \mathbf{T}(F)$, coming from $\alpha^{\vee}: \mathbb{G}_{m} \rightarrow \mathbf{T}$. Also $\pi_{v}$ is a uniformiser of $F_{v}$, viewed as an element of $\mathbb{A}_{F}^{*}$.

The following proposition is obvious from the definitions.

Proposition 7.23.

$$
\operatorname{Eis}_{\chi, P}=L_{P}(\chi) \operatorname{Eis}_{\chi}^{\prime}
$$

Remark 7.24. One can view Proposition 7.23 as the reason that $L$-function appears in the functional equation, satisfied by Eisenstein series (cf. [8]). Note that in the case when $F$ is a functional field and $\chi$ is everywhere unramified one can take $P=\emptyset$. In this case Eis $_{\chi, P}$ was constructed in [9], [2] and [1] by geometric methods. It would be interesting to understand a direct connection betweeen the construction in loc. cit. and the construction presented above.

\section{REFERENCES}

[1] A. Braverman and D. Gaitsgory, Geometric Eisenstein series, in preparation.

[2] D. Gaitsgory, Eisenstein series and automorphic sheaves for $G L(n), \mathrm{PhD}$. thesis, Tel-Aviv University, 1997.

[3] V. Ginzburg, M. Kapranov and E. Vasserot, Residue construction of Hecke algebras, Adv. Math. 128 (1997) 1-19.

[4] B. Feigin, M. Finkelberg, A. Kuznetsov and I. Mirkovic, Semiinfinite Flags II

[5] S.-I. Kato, On the Kazhdan-Lusztig polynomials for affine Weyl groups, Adv. in Math. 55, 103130 (1985).

[6] D. Kazhdan, "Forms" of the principle series for $G L_{n}$, in: Functional analysis on the Eve of the 21-st century, Progress in Math., Birkhäuser, 131 (1995) 153-172

[7] D. Kazhdan, G. Lusztig, Proof of the Deligne-Langlands conjecture for Hecke algebras, Invent. Math. 87 (1987) 153-215. 
[8] R. P. Langlands, On the functional equations satisfied by Eisenstein series, Lecture Notes in Mathematics, 544 Springer-Verlag, Berlin-New York, 1976.

[9] G. Laumon, Faisceaux automorphes liés aux séries d'Eisenstein (French) [Automorphic sheaves associated with Eisenstein series] Automorphic forms, Shimura varieties, and $L$-functions, Vol. I (Ann Arbor, MI, 1988), 227-281, Perspect. Math., 10, Academic Press, Boston, MA, 1990.

[10] G. Lusztig, Hecke algebras and Jantzen's generic decomposition patterns, Adv. in Math. 37 (1980), no. 2, 121-164.

[11] G. Lusztig, Periodic W-graphs, Represent. Theory 1 (1997), 207-279 (electronic).

[12] G. Lusztig, Bases in equivariant K-theory, Preprint.

[13] A. Weil, Adéles and algebraic groups, Progr. in Math., Birkhäuser, Boston 1982.

2-175, Department of Mathematics, Massachusetts Institute of Technology 77 Massachusetts Ave., Cambridge MA, USA

Department of Mathematics Harvard University 1 Oxford st. Cambridge MA, USA

E-mail address: braval@math.mit.edu, kazhdan@math.harvard.edu 